Document downloaded from:

http://hdl.handle.net/10251/103256

This paper must be cited as:

Bayart, F.; Defant, A.; Frerick, L.; Maestre, M.; Sevilla Peris, P. (2017). Multipliers of Dirichlet series and monomial series expansions of holomorphic functions in infinitely many variables. Mathematische Annalen. 368(1-2):837-876. doi:10.1007/s00208-016-1511-1

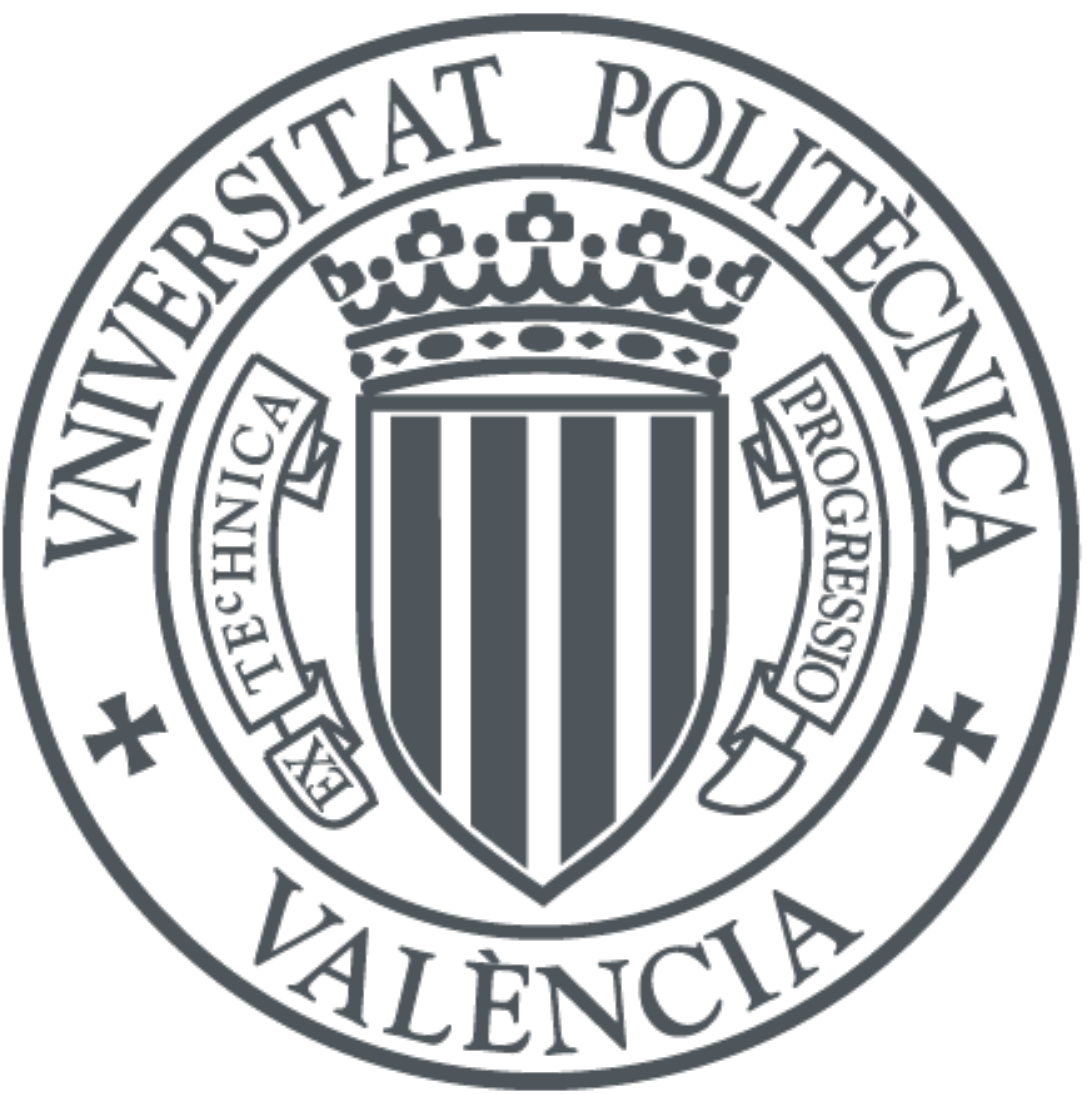

The final publication is available at

https://doi.org/10.1007/s00208-016-1511-1

Copyright Springer-Verlag

Additional Information 


\title{
Multipliers of Dirichlet series and monomial series expansions of holomorphic functions in infinitely many variables
}

\author{
Frédéric Bayart * Andreas Defant ${ }^{\dagger} \quad$ Leonhard Frerick $^{\ddagger}$ \\ Manuel Maestre ${ }^{\S} \quad$ Pablo Sevilla-Peris `
}

\begin{abstract}
Let $\mathscr{H}_{\infty}$ be the set of all ordinary Dirichlet series $D=\sum_{n} a_{n} n^{-s}$ representing bounded holomorphic functions on the right half plane. A multiplicative sequence $\left(b_{n}\right)$ of complex numbers is said to be an $\ell_{1}$-multiplier for $\mathscr{H}_{\infty}$ whenever $\sum_{n}\left|a_{n} b_{n}\right|<\infty$ for every $D \in \mathscr{H}_{\infty}$. We study the problem of describing such sequences $\left(b_{n}\right)$ in terms of the asymptotic decay of the subsequence $\left(b_{p_{j}}\right)$, where $p_{j}$ denotes the $j$ th prime number. Given a multiplicative sequence $b=\left(b_{n}\right)$ we prove (among other results): $b$ is an $\ell_{1}$ multiplier for $\mathscr{H}_{\infty}$ provided $\left|b_{p_{j}}\right|<1$ for all $j$ and $\varlimsup_{n} \frac{1}{\log n} \sum_{j=1}^{n} b_{p_{j}}^{* 2}<1$, and conversely, if $b$ is an $\ell_{1}$-multiplier for $\mathscr{H}_{\infty}$, then $\left|b_{p_{j}}\right|<1$ for all $j$ and $\varlimsup_{n} \frac{1}{\log n} \sum_{j=1}^{n} b_{p_{j}}^{* 2} \leq 1$ (here $b^{*}$ stands for the decreasing rearrangement of $b$ ). Following an ingenious idea of Harald Bohr it turns out that this problem is intimately related with the question of characterizing those sequences $z$ in the infinite dimensional polydisk $\mathbb{D}^{\infty}$ (the open unit ball of $\ell_{\infty}$ ) for which every bounded and holomorphic function $f$ on $\mathbb{D}^{\infty}$ has an absolutely convergent monomial series expansion $\sum_{\alpha} \frac{\partial_{\alpha} f(0)}{\alpha !} z^{\alpha}$. Moreover, we study analogous problems in Hardy spaces of Dirichlet series and Hardy spaces of functions on the infinite dimensional polytorus $\mathbb{T}^{\infty}$.
\end{abstract}

${ }^{*}$ Laboratoire de Mathématiques Université Blaise Pascal Campus des Cézeaux, F-63177 Aubière Cedex (France)

${ }^{\dagger}$ Institut für Mathematik. Universität Oldenburg. D-26111 Oldenburg (Germany)

${ }^{\ddagger}$ Fachbereich IV - Mathematik, Universität Trier, D-54294 Trier (Germany)

${ }^{\S}$ Dep. Análisis Matemático. Fac. Matemáticas. Universidad de Valencia. 46100 Burjassot (Spain)

"IUMPA. Universitat Politècnica de València. 46022 València (Spain)

The second, fourth and fifth authors were supported by MICINN and FEDER Project MTM2011-22417. The fourth author was also supported by PrometeoII/2013/013. The fifth author was also supported by project SP-UPV20120700.

Mathematics Subject Classification (2010): 46E50, 42B30, 30B50, 46G25

Keywords: Dirichlet series, power series expansion; holomorphic function in infinitely many variables; Hardy spaces; multipliers; Bohr's problem 


\section{Contents}

\begin{tabular}{llr}
\hline & Introduction & 2
\end{tabular}

\begin{tabular}{|lll|}
\hline 2 & Monomial expansion of $\boldsymbol{H}_{\infty}$-functions in infinitely many variables & 7
\end{tabular}

2.1 Statement of the results $\ldots \ldots \ldots \ldots$

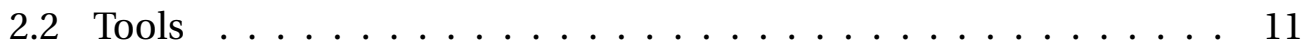

2.3 Proof of Theorem 2.1 Hower inclusion . . . . . . . . . . . . . 13

2.4 Proof of Theorem 2.2 Hower inclusion . . . . . . . . . . . . . . 14

2.5 The probabilistic device $\ldots \ldots \ldots \ldots \ldots$

2.6 Dismissing candidates . . . . . . . . . . . . . . . 21

3 Series expansion of $\boldsymbol{H}_{p}$-functions in infinitely many variables 23

3.1 The homogeneous case . . . . . . . . . . . . . 24

3.2 The general case . . . . . . . . . . . . . . . . . 27

3.3 Representation of Hardy spaces . . . . . . . . . . . . . . 33

\begin{tabular}{|lll}
4 & $\ell_{1}$-multipliers of $\mathscr{H}_{p}$-Dirichlet series & 35
\end{tabular}

4.1 Main results $\ldots \ldots \ldots \ldots \ldots \ldots$

4.2 Bohr's absolute convergence problem - old art in new light . . . . 38

\section{Introduction}

Recall from [11] that the precise asymptotic order of the Sidon constant of all finite Dirichlet polynomials $\sum_{n=1}^{N} a_{n} n^{-s}$ is given by

$$
\sup _{a_{1}, \ldots, a_{N} \in \mathbb{C}} \frac{\sum_{n=1}^{N}\left|a_{n}\right|}{\sup _{t \in \mathbb{R}} \mid \sum_{n=1}^{N} a_{n} n^{-i t \mid}}=\frac{\sqrt{N}}{e^{\frac{1}{\sqrt{2}}(1+o(1)) \sqrt{\log N \log \log N}}} .
$$

This result has its origin in fundamental works of Hilbert [21], Bohr [6], Toeplitz [28] and Bohnenblust-Hille [5], and it is the final outcome of a long series of results, beginning with the papers [25] of Queffélec and [23] of Konyagin-Queffélec which were later followed by [10] and [11]; for a complete presentation of the proof see the recent monograph [26].

As usual we denote by $\mathscr{H}_{\infty}$ the vector space of all ordinary Dirichlet series $\sum_{n} a_{n} n^{-s}$ representing bounded holomorphic functions on the right half plane (which together with the sup norm forms a Banach space). Applying (1) to dyadic blocks, it was proved in [11] (completing earlier results from [2]) that the supremum over all $c>0$ for which

$$
\sum_{n=1}^{\infty}\left|a_{n}\right| \frac{e^{c \sqrt{\log n \log \log n}}}{n^{\frac{1}{2}}}<\infty \text { for all } \sum_{n} a_{n} n^{-s} \in \mathscr{H}_{\infty}
$$


equals $1 / \sqrt{2}$. In other terms, all sequences

$$
\left(b_{n}\right)=\left(e^{(1 / \sqrt{2}-\varepsilon) \sqrt{\log n \log \log n}} n^{-1 / 2}\right), 0<\varepsilon<1 / \sqrt{2}
$$

are $\ell_{1}$-multipliers of $\mathscr{H}_{\infty}$ in the sense that

$$
\sum_{n=1}^{\infty}\left|a_{n} b_{n}\right|<\infty \text { for all } \sum_{n} a_{n} n^{-s} \in \mathscr{H}_{\infty} .
$$

Recall that a sequence $\left(b_{n}\right)$ is said to be (completely) multiplicative whenever $b_{n m}=b_{n} b_{m}$ for all $n, m$, and (2) obviously shows that the sequence $(1 / \sqrt{n})$ is a multiplicative $\ell_{1}$-multiplier of $\mathscr{H}_{\infty}$. Clearly, there are more such multiplicative $\ell_{1}$-multipliers of $\mathscr{H}_{\infty}$. For example, it will turn out that all multiplicative sequences $\left(b_{n}\right)$ with $\left|b_{n}\right|<1$ for all $n$ and such that $b_{p_{j}}=0$ for all but finitely many $j$ have this property; here as usual $p=\left(p_{j}\right)=\{2,3,5 \ldots\}$ stands for the sequence of primes.

In this article we intend to study the problem of describing all multiplicative $\ell_{1}$-multipliers $\left(b_{n}\right)$ of $\mathscr{H}_{\infty}$ in terms of the asymptotic decay of the subsequence $\left(b_{p_{j}}\right)$.

Surprisingly, this question is intimately related with the following natural problem: Do $\mathbb{C}$-valued holomorphic functions on the infinite dimensional polydisk $B_{\ell_{\infty}}$ (the open unit ball of the Banach space $\ell_{\infty}$ of all bounded scalar sequences), like in finite dimensions, have a reasonable monomial series expansion?

The crucial link is due to a brillant observation of Harald Bohr from [6] which we explain now: Denote by $\mathfrak{P}$ the vector space of all formal power series $\sum_{\alpha} c_{\alpha} z^{\alpha}$, and by $\mathfrak{D}$ the vector space of all Dirichlet series $\sum a_{n} n^{-s}$. By the fundamental theorem of arithmetic each $n \in \mathbb{N}$ has a unique prime decomposition $n=p^{\alpha}=p_{1}^{\alpha_{1}} \cdots p_{k}^{\alpha_{k}}$ with a multiindex $\alpha \in \mathbb{N}_{0}^{(\mathbb{N})}$ (i.e., $\alpha$ is a finite sequences of elements $\alpha_{k} \in \mathbb{N}_{0}$ ). Then the so-called Bohr transform $\mathfrak{B}$ is a linear algebra homomorphism:

$$
\mathfrak{B}: \mathfrak{P} \longrightarrow \mathfrak{D}, \quad \sum_{\alpha \in \mathbb{N}_{0}^{(\mathbb{N})}} c_{\alpha} z^{\alpha} \rightsquigarrow \sum_{n=1}^{\infty} a_{n} n^{-s} \text { with } a_{p^{\alpha}}=c_{\alpha} .
$$

Hilbert in [21] was among the very first who started a systematic study of the concept of analyticity for functions in infinitely many variables. According to Hilbert, an analytic function in infinitely many variables is a $\mathbb{C}$-valued function defined on the infinite dimensional polydisk $B_{\ell_{\infty}}$ (see above) which has a pointwise convergent monomial series expansion:

$$
f(z)=\sum_{\alpha \in \mathbb{N}_{0}^{(\mathbb{N})}} c_{\alpha} z^{\alpha}, z \in B_{\ell_{\infty}} .
$$


In [21] (see also [20, p. 65]) he gave the following criterion for a formal power series $\sum_{\alpha} c_{\alpha} z^{\alpha}$ to generate such a function (i.e., to converge absolutely at each point of $B_{\ell_{\infty}}$ ): Every $k$-dimensional section $\sum_{\alpha \in \mathbb{N}_{0}^{k}} c_{\alpha} z^{\alpha}$ of the series is pointwise convergent on $\mathbb{D}^{k}$, and moreover

$$
\sup _{k \in \mathbb{N}} \sup _{z \in \mathbb{D}^{k}}\left|\sum_{\alpha \in \mathbb{N}_{0}^{k}} c_{\alpha} z^{\alpha}\right|<\infty .
$$

But this criterion is not correct as was later discovered by Toeplitz (see below (8)). Why?

Today a holomorphic function $f: B_{\ell_{\infty}} \rightarrow \mathbb{C}$ is nothing but a Fréchet differentiable function $f: B_{\ell_{\infty}} \rightarrow \mathbb{C}$. As usual the Banach space of all bounded holomorphic $f: B_{\ell_{\infty}} \rightarrow \mathbb{C}$ endowed with the supremum norm will be denoted by $H_{\infty}\left(B_{\ell_{\infty}}\right)$. Important examples of such functions are bounded $m$-homogeneous polynomials $P: \ell_{\infty} \rightarrow \mathbb{C}$, restrictions of bounded $m$-linear forms on $\ell_{\infty} \times \cdots \times \ell_{\infty}$ to the diagonal. The vector space $\mathscr{P}\left({ }^{m} \ell_{\infty}\right)$ of all such $P$ together with the norm $\|P\|=\sup _{z \in B_{\ell_{\infty}}}|P(z)|$ forms a closed subspace of $H_{\infty}\left(B_{\ell_{\infty}}\right)$.

From the theory in finitely many variables it is well known that every holomorphic $\mathbb{C}$-valued mapping $f$ on the $k$-dimensional polydisk $\mathbb{D}^{k}$ has a monomial series expansion which converges to $f$ at every point of $\mathbb{D}^{k}$. More precisely, for every such $f$ there is a unique family $\left(c_{\alpha}(f)\right)_{\alpha \in \mathbb{N}_{0}^{k}}$ in $\mathbb{C}$ such that $f(z)=$ $\sum_{\alpha \in \mathbb{N}_{0}^{k}} c_{\alpha}(f) z^{\alpha}$ for every $z \in \mathbb{D}^{k}$. The coefficients can be calculated through the Cauchy integral formula or partial derivatives: For each $0<r<1$ and each $\alpha$

$$
c_{\alpha}(f)=\frac{\partial^{\alpha} f(0)}{\alpha !}=\frac{1}{(2 \pi i)^{k}} \int_{\left|z_{1}\right|=r} \ldots \int_{\left|z_{k}\right|=r} \frac{f(z)}{z^{\alpha+1}} d z_{1} \ldots d z_{k} .
$$

Clearly, every holomorphic function $f: B_{\ell_{\infty}} \rightarrow \mathbb{C}$, whenever restricted to a finite dimensional section $\mathbb{D}^{k}=\mathbb{D}^{k} \times\{0\}$, has an everywhere convergent power series expansion $\sum_{\alpha \in \mathbb{N}_{0}^{k}} c_{\alpha}^{(k)}(f) z^{\alpha}, z \in \mathbb{D}^{k}$. And from (7) we see that $c_{\alpha}^{(k)}(f)=c_{\alpha}^{(k+1)}(f)$ for $\alpha \in \mathbb{N}_{0}^{k} \subset \mathbb{N}_{0}^{k+1}$. Thus again there is a unique family $\left(c_{\alpha}(f)\right)_{\alpha \in \mathbb{N}_{0}^{(\mathbb{N})}}$ in $\mathbb{C}$ such that at least for all $k \in \mathbb{N}$ and all $z \in \mathbb{D}^{k}$

$$
f(z)=\sum_{\alpha \in \mathbb{N}_{0}^{(\mathbb{N})}} c_{\alpha}(f) z^{\alpha} .
$$

This power series is called the monomial series expansion of $f$, and $c_{\alpha}=c_{\alpha}(f)$ are its monomial coefficients; by definition they satisfy (7) whenever $\alpha \in \mathbb{N}_{0}^{k}$.

At first one could expect that each $f \in H_{\infty}\left(B_{\ell_{\infty}}\right)$ has a monomial series expansion which again converges at every point and represents the function. But this is not the case: Just take a non-zero functional on $\ell_{\infty}$ that is 0 on $c_{0}$ (the 
space of null sequences); by definition, its monomial series expansion is 0 and clearly does not represent the function. Moreover, since such a functional obviously satisfies (6), although it is not analytic (in Hilbert's sense), the criterion of Hilbert turns out to be false.

In order to avoid this example one could now try with the open unit ball $B_{c_{0}}$ of $c_{0}$ instead of $B_{\ell_{\infty}}$. But Hilbert's criterion remains false: Note first that a simple extension argument (see e.g. [12, Lemma 2.2]) allows to identify all formal power series satisfying (6) with all bounded holomorphic functions on $B_{c_{0}}$; more precisely, each $f \in H_{\infty}\left(B_{c_{0}}\right)$ has a monomial series expansion as in (6), and conversely each power series satisfying (6) gives rise to a unique $f \in$ $H_{\infty}\left(B_{c_{0}}\right)$ for which $c_{\alpha}=c_{\alpha}(f)$ for all $\alpha$.

But then (6) does not imply (5) since by an example of Toeplitz from [28] there is a 2-homogeneous bounded polynomial $P$ on $c_{0}$ such that

$$
\forall \varepsilon>0 \exists x \in \ell_{4+\varepsilon}: \sum_{\alpha}\left|c_{\alpha}(P) x^{\alpha}\right|=\infty
$$

This means that there are functions $f \in H_{\infty}\left(B_{c_{0}}\right)$ that cannot be pointwise described by their monomial series expansions as in (5) which, at least at first glance, seems disillusioning. Indeed, this fact in infinite dimensions produces a sort of dilemma: There is no way to develop a complex analysis of functions in infinitely many variables which simultaneously handles phenomena on differentiability and analyticity (as it happens in finite dimensions).

One of the main advances of this article is to give an almost complete description of what we call the set of monomial convergence of all bounded holomorphic functions on the open unit ball $B_{\ell_{\infty}}$ of $\ell_{\infty}$ :

$$
\operatorname{mon} H_{\infty}\left(B_{\ell_{\infty}}\right)=\left\{z \in B_{\ell_{\infty}} \mid \forall f \in H_{\infty}\left(B_{\ell_{\infty}}\right): f(z)=\sum_{\alpha \in \mathbb{N}_{0}^{(\mathbb{N})}} c_{\alpha}(f) z^{\alpha}\right\} .
$$

We recall that the decreasing rearrangement of $z \in \ell_{\infty}$ is given by

$$
z_{n}^{*}=\inf \left\{\sup _{j \in \mathbb{N} \backslash J}\left|z_{j}\right| \mid J \subset \mathbb{N}, \operatorname{card}(J)<n\right\},
$$

and use it to define the set

$$
\mathbf{B}=\left\{z \in B_{\ell_{\infty}} \mid \boldsymbol{b}(z)=\left(\limsup _{n \rightarrow \infty} \frac{1}{\log n} \sum_{j=1}^{n} z_{j}^{* 2}\right)^{1 / 2}<1\right\} .
$$

Then our main result on sets of monomial convergence is Theorem 2.2 that shows

$$
\mathbf{B} \subset \operatorname{mon} H_{\infty}\left(B_{\ell_{\infty}}\right) \subset \overline{\mathbf{B}} .
$$


As we intend to indicate in the following sections, this result has a long list of forerunners (due to various authors, see e.g. [5, 6, 7, 8, 14, 20, 21, 28]). In (17), (18), (20), (21), (22) as well as (49), (50), (52), (53) it will become clear that mon $H_{\infty}\left(B_{\ell_{\infty}}\right)$ was known to be very close to $\ell_{2} \cap B_{\ell_{\infty}}$. But (11) adds a new level of precision that enables us to extract much more precise information from monomial convergence of holomorphic functions on the infinite dimensional polydisk than before.

This in particular becomes clear if we finally return to the beginning of this introduction - let us return to the description of all multiplicative $\ell_{1}$-multipliers of $\mathscr{H}_{\infty}$ using Bohr's transform from (4). The following fact, essentially due to Bohr [6] and later rediscovered in [18, Lemma 2.3 and Theorem 3.1], is essential: The Bohr transform $\mathfrak{B}$ induces an isometric algebra isomorphism from $H_{\infty}\left(B_{c_{0}}\right)$ onto $\mathscr{H}_{\infty}$,

$$
H_{\infty}\left(B_{c_{0}}\right)=\mathscr{H}_{\infty}
$$

This identification in fact allows to identify the multiplicative $\ell_{1}$-multipliers of $\mathscr{H}_{\infty}$ with the elements in mon $H_{\infty}\left(B_{\ell_{\infty}}\right)$ : Given a multiplicative sequence $\left(b_{n}\right) \in$ $\mathbb{C}^{\mathbb{N}}$, we have that

$$
\left(b_{n}\right) \text { is an } \ell_{1} \text {-multiplier for } \mathscr{H}_{\infty} \Leftrightarrow\left(b_{p_{k}}\right) \in \operatorname{mon} H_{\infty}\left(B_{\ell_{\infty}}\right) \text {. }
$$

Observe that this way we may deduce from (2) that the sequence

$$
\left(1 / \sqrt{p_{k}}\right) \in \operatorname{mon} H_{\infty}\left(B_{\ell_{\infty}}\right)
$$

this seems to be the first non-trivial example which distinguishes mon $H_{\infty}\left(B_{\ell_{\infty}}\right)$ from $\ell_{2} \cap B_{\ell_{\infty}}$. But note that this can also be seen using (11); indeed, (13) is a very particular case of the formulation of (11) given in Section 4 below.

Our main result is an almost complete characterization of all multiplicative $\ell_{1}$-multipliers for $\mathscr{H}_{\infty}$ (recall (10) for the definition of $\boldsymbol{b}(\cdot)$ ): For all multiplicative sequences $\left(b_{n}\right) \in \mathbb{C}^{\mathbb{N}}$ we have that

- $\left(b_{n}\right)$ is an $\ell_{1}$-multiplier for $\mathscr{H}_{\infty}$ provided we have that $\left|b_{p_{j}}\right|<1$ for all $j$ and $\boldsymbol{b}\left(\left(b_{p_{j}}\right)\right)<1$, and conversely,

- if $\left(b_{n}\right)$ is $\ell_{1}$-multiplier for $\mathscr{H}_{\infty}$, then $\left|b_{p_{j}}\right|<1$ for all $j$ and $\boldsymbol{b}\left(\left(b_{p_{j}}\right)\right) \leq 1$.

In Section 3 we extend our concept on sets of monomial convergence to $H_{p^{-}}$ functions defined on the infinite dimension torus $\mathbb{T}^{\infty}$ (see (34) for the precise definition); here $\mathbb{T}$ denotes the torus (the unit circle of $\mathbb{C}$ ) and $\mathbb{T}^{\infty}$ the infinite dimensional polytorus (the countable cartesian product of $\mathbb{T}$ ). The Banach space $H_{\infty}\left(B_{c_{0}}\right)$ can be isometrically identified with the Banach space $H_{\infty}\left(\mathbb{T}^{\infty}\right)$ of all 
$L_{\infty}$-functions $f: \mathbb{T}^{\infty} \rightarrow \mathbb{C}$ with Fourier coefficients $\hat{f}(\alpha)=0$ for $\alpha \in \mathbb{Z}^{(\mathbb{N})} \backslash \mathbb{N}_{0}^{(\mathbb{N})}$; this was proved in [8] (see also Proposition 3.5). For $1 \leq p \leq \infty$ we define

$$
\begin{aligned}
H_{p}\left(\mathbb{T}^{\infty}\right) & =\left\{f \in L_{p}\left(\mathbb{T}^{\infty}\right) \mid \hat{f}(\alpha)=0, \forall \alpha \in \mathbb{Z}^{(\mathbb{N})} \backslash \mathbb{N}_{0}^{(\mathbb{N})}\right\} \\
\operatorname{mon} H_{p}\left(\mathbb{T}^{\infty}\right) & =\left\{z \in \mathbb{C}^{\mathbb{N}}\left|\sum_{\alpha}\right| \hat{f}(\alpha) z^{\alpha} \mid<\infty \text { for all } f \in H_{p}\left(\mathbb{T}^{\infty}\right)\right\} .
\end{aligned}
$$

Then it is not hard to see that mon $H_{\infty}\left(\mathbb{T}^{\infty}\right)=$ mon $H_{\infty}\left(B_{\ell_{\infty}}\right)$, but in contrast to (11) we have

$$
\operatorname{mon} H_{p}\left(\mathbb{T}^{\infty}\right)=\ell_{2} \cap B_{\ell_{\infty}} \text { for all } 1 \leq p<\infty .
$$

This way we extend and complement results of Cole and Gamelin from [8]. Finally, in Section 4 we use Bohr's vision from (4) to interpret all these results on sets of monomial convergence of $H_{p}$-functions in terms of multiplicative $\ell_{1}$ multipliers for $\mathscr{H}_{p}$-Dirichlet series (as was already described above for the case $p=\infty)$.

\section{Monomial expansion of $\boldsymbol{H}_{\infty}$-functions in infinitely many variables}

Our definition of sets of monomial convergence (9) has its roots in Bohr's seminal article [6], and the first systematic study of such sets was undertaken in [14]. Recall from the introduction that

$$
\operatorname{mon} H_{\infty}\left(B_{\ell_{\infty}}\right)=\left\{z \in B_{\ell_{\infty}} \mid \forall f \in H_{\infty}\left(B_{\ell_{\infty}}\right): f(z)=\sum_{\alpha \in \mathbb{N}_{0}^{(\mathbb{N})}} c_{\alpha}(f) z^{\alpha}\right\},
$$

and define similarly for $m \in \mathbb{N}$

$$
\operatorname{mon} \mathscr{P}\left({ }^{m} \ell_{\infty}\right)=\left\{z \in \ell_{\infty} \mid \forall P \in \mathscr{P}\left({ }^{m} \ell_{\infty}\right): P(z)=\sum_{\alpha \in \mathbb{N}_{0}^{(\mathbb{N})}} c_{\alpha}(P) z^{\alpha}\right\}
$$

Since we here consider functions $f$ defined on $B_{\ell_{\infty}}$ as well as polynomials $P$ defined on $\ell_{\infty}$, we clearly cannot avoid to define the preceding two sets as subsets of $B_{\ell_{\infty}}$ and $\ell_{\infty}$, respectively. Nevertheless we can give two slight reformulations which will be of particular importance when we translate our forthcoming results into terms of multipliers for Dirichlet series:

$$
\operatorname{mon} H_{\infty}\left(B_{\ell_{\infty}}\right)=\left\{z \in \mathbb{C}^{\mathbb{N}}\left|\forall f \in H_{\infty}\left(B_{\ell_{\infty}}\right): \sum_{\alpha \in \mathbb{N}_{0}^{(\mathbb{N})}}\right| c_{\alpha}(f) z^{\alpha} \mid<\infty\right\}
$$




$$
\operatorname{mon} \mathscr{P}\left({ }^{m} \ell_{\infty}\right)=\left\{z \in \mathbb{C}^{\mathbb{N}}\left|\forall P \in \mathscr{P}\left({ }^{m} \ell_{\infty}\right): \sum_{\alpha \in \mathbb{N}_{0}^{(\mathbb{N})}}\right| c_{\alpha}(P) z^{\alpha} \mid<\infty\right\}
$$

The argument for these two equalities is short: Denote the set in (15) by $U$, and that in (14) by $V$. For $z \in U$ it was shown in [14, p.29-30] that $z \in c_{0}$. Then an obvious continuity argument gives the equality in (15). Take now $z \in V \subset U$. Considering bounded holomorphic functions on the open disk $\mathbb{D}$, we see immediately that $\left|z_{n}\right|<1$ for all $n$. The equality in (14) again follows by continuity.

In the above definitions we may replace $\ell_{\infty}$ by $c_{0}$. Davie and Gamelin showed in [9, Theorem 5] that every function in $H_{\infty}\left(B_{c_{0}}\right)$ can be extended to a function in $H_{\infty}\left(B_{\ell_{\infty}}\right)$ with the same norm. Using this it was shown in [14, Remark 6.4] that we in fact have

$$
\operatorname{mon} H_{\infty}\left(B_{\ell_{\infty}}\right)=\operatorname{mon} H_{\infty}\left(B_{c_{0}}\right) \text { and } \operatorname{mon} \mathscr{P}\left({ }^{m} \ell_{\infty}\right)=\operatorname{mon} \mathscr{P}\left({ }^{m} c_{0}\right) \text {. }
$$

Let us collect some more basic facts on sets of monomial convergence which in the following will be used without further reference:

- If $z \in$ mon $H_{\infty}\left(B_{\ell_{\infty}}\right)$, then every permutation of $z$ is again in mon $H_{\infty}\left(B_{\ell_{\infty}}\right)$; this was proved in [13, p. 550].

- We know from [14, p. 29-30] that mon $H_{\infty}\left(B_{\ell_{\infty}}\right) \subset c_{0}$. Hence, provided $z \in$ mon $H_{\infty}\left(B_{\ell_{\infty}}\right)$ does not have a finite support, there exists a bijection from $\mathbb{N}$ onto the support of $z$ such that $z^{*}=\left(\left|z_{\sigma(n)}\right|\right)$. This implies that $z \in \operatorname{mon} H_{\infty}\left(B_{\ell_{\infty}}\right)$ if and only if $z^{*} \in \operatorname{mon} H_{\infty}\left(B_{\ell_{\infty}}\right)$.

- Let $z \in$ mon $H_{\infty}\left(B_{\ell_{\infty}}\right)$ and $x=\left(x_{n}\right)_{n} \in B_{\ell_{\infty}}$ such that $\left|x_{n}\right| \leq\left|z_{n}\right|$ for all but finitely many $n$ 's. Then $x \in$ mon $H_{\infty}\left(B_{\ell_{\infty}}\right)$; this result is from [13, Lemma 2] and was inspired by [6, Satz VI] (see also Lemma3.7).

- Similar results hold for mon $\mathscr{P}\left({ }^{m} \ell_{\infty}\right)$.

What was so far known on sets of monomial convergence? Bohr [6] proved

$$
\ell_{2} \cap B_{\ell_{\infty}} \subset \operatorname{mon} H_{\infty}\left(B_{\ell_{\infty}}\right)
$$

and Bohnenblust-Hille in [5]

$$
\ell_{\frac{2 m}{m-1}} \subset \operatorname{mon} \mathscr{P}\left({ }^{m} \ell_{\infty}\right) .
$$

Moreover, these two results in a certain sense are optimal; to see this define

$$
\begin{aligned}
M & :=\sup \left\{1 \leq p \leq \infty \mid \ell_{p} \cap B_{\ell_{\infty}} \subset \operatorname{mon} H_{\infty}\left(B_{\ell_{\infty}}\right)\right\}, \\
M_{m} & :=\sup \left\{1 \leq p \leq \infty \mid \ell_{p} \subset \operatorname{mon} \mathscr{P}\left({ }^{m} \ell_{\infty}\right)\right\} \text { for } m \in \mathbb{N} .
\end{aligned}
$$


These are two quantities which measure the size of both sets of convergence in terms of the largest possible slices $\ell_{p} \cap B_{\ell_{\infty}}$ included in them. The definition of $M$ (at least implicitly) appears in [6], and (17) of course gives that $M \geq 2$. The idea of graduating $M$ through $M_{m}$ appears first in Toeplitz' article [28]; clearly the estimate $M_{2} \leq 4$ is a reformulation of [8]. After Bohr's paper [6] the intensive search for the exact value of $M$ and $M_{m}$ was not succesful for more then 15 years. The final answer was given by Bohnenblust and Hille in [5], who were able to prove that

$$
M_{m}=\frac{2 m}{m-1} \quad \text { and } \quad M=\frac{1}{2} .
$$

Their original proofs of the upper bounds are clever and ingenious. Using modern techniques of probabilistic nature, different from the original ones, they were improved in [14, Example 4.9 and Example 4.6]:

$$
\ell_{2} \cap B_{\ell_{\infty}} \subset \operatorname{mon} H_{\infty}\left(B_{\ell_{\infty}}\right) \subset \bigcap_{\varepsilon>0} \ell_{2+\varepsilon}
$$

and

$$
\operatorname{mon} \mathscr{P}\left({ }^{m} \ell_{\infty}\right) \subset \ell_{\frac{2 m}{m-1}, \infty}
$$

Recall that for $1 \leq q<\infty$ the Marcinkiewicz space $\ell_{q, \infty}$ consists of those sequences $z$ for which $\sup _{n} z_{n}^{*} n^{1 / q}<\infty$ (and this supremum defines the norm of this Banach space). Clearly, $\ell_{q, \infty} \subset c_{0}$.

\subsection{Statement of the results}

We already mentioned in (13) that the left inclusion in (21) is strict. The aim of this section is to show that our two sets of monomial convergence can be 'squeezed' in a much more drastic way. Our first theorem gives a complete description of mon $\mathscr{P}\left({ }^{m} \ell_{\infty}\right)$ and extends all results on this set mentioned so far.

Theorem 2.1. Let $m \in \mathbb{N}$. Then

$$
\operatorname{mon} \mathscr{P}\left({ }^{m} \ell_{\infty}\right)=\ell_{\frac{2 m}{m-1}, \infty},
$$

and moreover there exists a constant $C>0$ such that for every $z \in \ell_{\frac{2 m}{m-1}, \infty}$ and every $P \in \mathscr{P}\left({ }^{m} \ell_{\infty}\right)$ we have

$$
\sum_{|\alpha|=m}\left|c_{\alpha}(P) z^{\alpha}\right| \leq C^{m}\|z\|^{m}\|P\| .
$$

where $\|z\|$ denotes the norm of $z$ in $\ell_{\frac{2 m}{m-1}, \infty}$. 
In view of Bohr's transform $\mathfrak{B}$ from (4) this theorem can be seen as a sort of polynomial counterpart of a recent result on $m$-homogeneous Dirichlet series. A Dirichlet series $\sum a_{n} n^{-s}$ is called $m$-homogeneous whenever $a_{n}=0$ for every $\Omega(n) \neq m$; following standard notation, for each $n \in \mathbb{N}$ we write $\Omega(n)=|\alpha|$ if $n=$ $p^{\alpha}$ (this counts the prime divisors of $n$, according to their multiplicity). By $\mathscr{H}_{\infty}^{m}$ we denote the closed subspace of all $m$-homogeneous Dirichlet series in the Banach space $\mathscr{H}_{\infty}$. Then the restriction of the isometric algebra isomorphism $\mathfrak{B}: H_{\infty}\left(B_{c_{0}}\right) \rightarrow \mathscr{H}_{\infty}$ from (12) defines an isometric and linear bijection:

$$
\mathscr{P}\left({ }^{m} c_{0}\right)=\mathscr{H}_{\infty}^{m} .
$$

The following estimate due to Balasubramanian, Calado and Queffélec [2, Theorem 1.4] is a homogeneous counterpart of (2) and of Theorem 2.1: For each $m \geq 1$ there exists $C_{m}>0$ such that for every $\sum a_{n} n^{-s} \in \mathscr{H}_{\infty}^{m}$ we have

$$
\sum_{n}\left|a_{n}\right| \frac{(\log n)^{\frac{m-1}{2}}}{n^{\frac{m-1}{2 m}}} \leq C_{m} \sup _{t \in \mathbb{R}}\left|\sum_{n} a_{n} n^{i t}\right|,
$$

and the parameter $\frac{m-1}{2}$ is optimal by [24, Theorem 3.1] (here, in contrast to [23), it seems unknown whether the constant $C_{m}$ is subexponential).

At least philosophically holomorphic functions can be viewed as polynomials of degree $m=\infty$. Hence it is not surprising that the complete characterization of mon $\mathscr{P}\left({ }^{m} \ell_{\infty}\right)$ from Theorem 2.1 improves (17) and even the highly non-trivial fact from (13): With

$$
\ell_{2,0}=\left\{z \in \ell_{\infty} \mid \lim _{n} z_{n}^{*} \sqrt{n}=0\right\}
$$

we have

$$
\ell_{2} \cap B_{\ell_{\infty}} \varsubsetneqq \ell_{2,0} \cap B_{\ell_{\infty}} \subset \operatorname{mon} H_{\infty}\left(B_{\ell_{\infty}}\right) ;
$$

note that by the prime number theorem $\left(p_{n}^{-1 / 2}\right) \in \ell_{2,0} \cap B_{\ell_{\infty}}$ while this sequence does not belong to $\ell_{2}$. We sketch the proof of (26): Since

$$
B_{\ell_{2, \infty}} \subset \bigcap_{m \in \mathbb{N}} B_{\ell} \frac{2 m}{m-1}, \infty
$$

by [23) and [14, Theorem 5.1] there exists an $r>0$ such that we have $r B_{\ell_{2, \infty}} \subset$ mon $H_{\infty}\left(B_{\ell_{\infty}}\right)$. Then we conclude that $\left(\frac{r}{\sqrt{n}}\right)_{n} \in \operatorname{mon} H_{\infty}\left(B_{\ell_{\infty}}\right)$ which easily gives that $z^{*} \in$ mon $H_{\infty}\left(B_{\ell_{\infty}}\right)$ for every $z \in \ell_{2,0} \cap B_{\ell_{\infty}}$. By the general remarks on mon from the beginning of this section this completes the proof of (26).

Improving (26) considerably, the following theorem is our main result on monomial convergence of bounded holomorphic functions on the infinite dimensional polydisk. It can be seen as the power series counterpart of (2), and in Section 4 we will see that it gives far reaching information on the general theory of Dirichlet series. 
Theorem 2.2. For each $z \in B_{\ell_{\infty}}$ the following two statements hold:

(a) If $\limsup _{n \rightarrow \infty} \frac{1}{\log n} \sum_{j=1}^{n} z_{j}^{* 2}<1$, then $z \in \operatorname{mon} H_{\infty}\left(B_{\ell_{\infty}}\right)$.

(b) If $z \in \operatorname{mon} H_{\infty}\left(B_{\ell_{\infty}}\right)$, then $\limsup _{n \rightarrow \infty} \frac{1}{\log n} \sum_{j=1}^{n} z_{j}^{* 2} \leq 1$; moreover, here the converse implication is false.

In the remaining part of this section, we prove Theorems 2.1 and 2.2. To do so, we need some more notation: Given $k, m \in \mathbb{N}$ we consider the following sets of indices

$$
\begin{aligned}
& \mathscr{M}(m, k)=\left\{\mathbf{j}=\left(j_{1}, \ldots, j_{m}\right) \mid 1 \leq j_{1}, \ldots, j_{m} \leq k\right\}=\{1, \ldots, k\}^{m} \\
& \mathscr{J}(m, k)=\left\{\mathbf{j} \in \mathscr{M}(m, k) \mid 1 \leq j_{1} \leq \cdots \leq j_{m} \leq k\right\} \\
& \Lambda(m, k)=\left\{\alpha \in \mathbb{N}_{0}^{k}|| \alpha \mid=\alpha_{1}+\cdots+\alpha_{k}=m\right\}
\end{aligned}
$$

An equivalence relation is defined in $\mathscr{M}(m, k)$ as follows: $\mathbf{i} \sim \mathbf{j}$ if there is a permutation $\sigma$ such that $i_{\sigma(r)}=j_{r}$ for all $r$. We write $|\mathbf{i}|$ for the cardinality of the equivalence class [i]. For each $\mathbf{i} \in \mathscr{M}(m, k)$ there is a unique $\mathbf{j} \in \mathscr{J}(m, k)$ such that $\mathbf{i} \sim \mathbf{j}$. On the other hand, there is a one-to-one relation between $\mathscr{J}(m, k)$ and $\Lambda(m, k)$ : Given $\mathbf{j}$, one can define $\alpha$ by doing $\alpha_{r}=\left|\left\{q \mid j_{q}=r\right\}\right|$; conversely, for each $\alpha$, we consider $\mathbf{j}_{\alpha}=\left(1, \ldots, \ldots, 1,2, \alpha_{2}, 2, \ldots, k, \alpha_{k}, k\right)$. Note that $\left|\mathbf{j}_{\alpha}\right|=\frac{m !}{\alpha !}$ for every $\alpha \in \Lambda(m, k)$. Taking this correspondence into account, the monomial series expansion of a polynomial $P \in \mathscr{P}\left({ }^{m} \ell_{\infty}^{k}\right)$ can be expressed in different ways (we write $c_{\alpha}=c_{\alpha}(P)$ )

$$
\sum_{\alpha \in \Lambda(m, k)} c_{\alpha} z^{\alpha}=\sum_{\mathbf{j} \in \mathscr{J}(m, k)} c_{\mathbf{j}} z_{\mathbf{j}}=\sum_{1 \leq j_{1} \leq \ldots \leq j_{m} \leq k} c_{j_{1} \ldots j_{m}} z_{j_{1}} \cdots z_{j_{m}}
$$

\subsection{Tools}

The proof of Theorem 2.1 and Theorem 2.2-(a) share some similarities. They need several lemmas. The first one is a Khinchine-Steinhaus type inequality for $m$-homogeneous polynomials on the $n$-dimensional torus $\mathbb{T}^{n}$ (see [3] and also [29]). Following [27] and [30] $m_{n}$ and $m$ will denote the product of the normalized Lebesgue measure respectively on $\mathbb{T}^{n}$ and $\mathbb{T}^{\infty}$ (i.e. the unique rotation invariant Haar measures).

Lemma 2.3. Let $1 \leq r \leq s<\infty$. Then for every $m$-homogeneous polynomial $P \in$ $\mathscr{P}\left({ }^{m} \mathbb{C}^{n}\right)$ we have

$$
\left(\int_{\mathbb{T}^{n}}|P(w)|^{s} d m_{n}(w)\right)^{1 / s} \leq \sqrt{\frac{s}{r}}^{m}\left(\int_{\mathbb{T}^{n}}|P(w)|^{r} d m_{n}(w)\right)^{1 / r} .
$$


The second lemma needed for the proof of Lemma 2.5 is the following hypercontractive Bohnenblust-Hille inequality for $m$-homogeneous polynomials on the $n$-dimensional torus. This was recently shown in [4], improving a result from [11].

Lemma 2.4. For every $\kappa>1$ there is a constant $C(\kappa)>0$ such that for every $m$ homogeneous polynomial $P=\sum_{|\alpha|=m} c_{\alpha} z^{\alpha}, z \in \mathbb{C}^{n}$ we have

$$
\left(\sum_{\alpha \in \Lambda(m, n)}\left|c_{\alpha}\right|^{\frac{2 m}{m+1}}\right)^{\frac{m+1}{2 m}} \leq C(\kappa) \kappa^{m}\|P\|
$$

We are now ready to give the main technical tool.

Lemma 2.5. For every $\kappa>1$ and $p \in \mathbb{N}$ there is a constant $C(\kappa, p)>0$ such that for every $m \geq p$ and $P \in \mathscr{P}\left({ }^{m} \ell_{\infty}^{n}\right)$ with coefficients $\left(c_{\mathbf{j}}\right)_{\mathbf{j}}$, we have

$$
\left[\sum_{\mathbf{j} \in \mathscr{J}(p, n)}\left(\sum_{\substack{\mathbf{i} \in \mathscr{L}(m-p, n) \\ i_{m-p} \leq j_{1}}}\left|c_{(\mathbf{i}, \mathbf{j})}\right|^{2}\right]^{\frac{1}{2} \times \frac{2 p}{p+1}} \leq C(\kappa, p)\left[\kappa\left(1+\frac{1}{p}\right)\right]^{\frac{p+1}{2 p}}\|P\| .\right.
$$

Proof. Let us start by denoting

$$
H:=\left[\sum_{\mathbf{j} \in \mathscr{J}(p, n)}\left(\sum_{\substack{\mathbf{i} \in \mathscr{J}(m-p, n) \\ i_{m-p} \leq j_{1}}}\left|c_{(\mathbf{i}, \mathbf{j})}\right|^{2}\right)^{\frac{1}{2} \times \frac{2 p}{p+1}}\right]^{\frac{p+1}{2 p}} .
$$

Let $L$ be the symmetric $m$-linear form associated to $P$, whose coefficients $a_{i_{1}, \ldots, i_{m}}=$ $L\left(e_{i_{1}}, \ldots, e_{i_{m}}\right)$ satisfy, for $\mathbf{i} \in \mathscr{J}(m, n)$,

$$
a_{\mathbf{i}}=\frac{c_{\mathbf{i}}}{|\mathbf{i}|}
$$

We fix $\mathbf{j} \in \mathscr{J}(p, n)$, and note that for any $\mathbf{i} \in \mathscr{J}(m-p, n)$

$$
|(\mathbf{i}, \mathbf{j})| \leq m(m-1) \cdots(m-p+1)|\mathbf{i}| .
$$

Then

$$
\sum_{\substack{\mathbf{i} \in \mathscr{\mathscr { J }}(m-p, n) \\ i_{m-p} \leq j_{1}}}\left|c_{(\mathbf{i}, \mathbf{j})}\right|^{2} \leq \sum_{\mathbf{i} \in \mathscr{J}(m-p, n)}|(\mathbf{i}, \mathbf{j})|^{2}\left|a_{(\mathbf{i}, \mathbf{j})}\right|^{2} \leq m^{2 p} \sum_{\mathbf{i} \in \mathscr{J}(m-p, n)}|\mathbf{i}|^{2}\left|a_{(\mathbf{i}, \mathbf{j})}\right|^{2} .
$$


We now apply Lemma 2.3 with the exponent $\frac{2 p}{p+1}$ to the $(m-p)$-homogeneous polynomial $z \mapsto L\left(z, \ldots, z, e_{j_{1}}, \ldots, e_{j_{p}}\right)$ to get

$$
\begin{aligned}
\sum_{\substack{\mathbf{i} \in \mathscr{J}(m-p, n) \\
i_{m-p} \leq j_{1}}}\left|c_{(\mathbf{i}, \mathbf{j})}\right|^{2} \leq & m^{2 p}\left(1+\frac{1}{p}\right)^{m} \\
& \times\left(\int_{\mathbb{T}^{n}}\left|\sum_{\mathbf{i} \in \mathscr{L}(m-p, n)}\right| \mathbf{i}\left|a_{(\mathbf{i}, \mathbf{j})} w_{i_{1}} \cdots w_{i_{m-p}}\right|^{\frac{2 p}{p+1}} d m_{n}(w)\right)^{\frac{p+1}{2 p} \times 2} .
\end{aligned}
$$

We then sum over $\mathbf{j} \in \mathscr{J}(p, n)$. This yields

$$
H^{\frac{2 p}{p+1}} \leq m^{\frac{(2 p)^{2}}{2(p+1)}}\left(1+\frac{1}{p}\right)^{m \times \frac{2 p}{p+1}} \times \int_{\mathbb{T}^{n}} \sum_{\mathbf{j} \in \mathcal{F}(p, n)} \mid L\left(w, \ldots, w, e_{j_{1}}, \ldots, e_{j_{p}}\right)^{\frac{2 p}{p+1}} d m_{n}(w) .
$$

For each fixed $w \in \mathbb{T}^{n}$ we apply Lemma 2.4 with $1<\kappa_{0}<\kappa$ to the $p$-homogeneous polynomial $z \mapsto L(w, \ldots, w, z, \ldots, z)$ :

$$
\begin{aligned}
H^{\frac{2 p}{p+1}} & \leq C\left(\kappa_{0}\right) m^{\frac{(2 p)^{2}}{2(p+1)}}\left[\left(1+\frac{1}{p}\right) \kappa_{0}\right]^{m \times \frac{2 p}{p+1}} \sup _{w, z \in \mathbb{T}^{n}}|L(w, \ldots, w, z, \ldots, z)|^{\frac{2 p}{p+1}} \\
& \leq C^{\prime}\left(\kappa_{0}\right) m^{\frac{(2 p)^{2}}{2(p+1)}}\left[\left(1+\frac{1}{p}\right) \kappa_{0}\right]^{m \times \frac{2 p}{p+1}} m^{\frac{p}{p+1}}\|P\|^{\frac{2 p}{p+1}}
\end{aligned}
$$

where in the last estimate we have used an inequality from Harris [17, Theorem 1].

\subsection{Proof of Theorem 2.1-lower inclusion}

Let $z \in \ell_{\frac{2 m}{m-1}, \infty}$, so that $\sup _{n} z_{n}^{*} n^{\frac{m-1}{2 m}}=\|z\|<\infty$. Let us fix $n \geq 1$ and let us consider $P \in \mathscr{P}\left({ }^{m} \ell_{\infty}^{n}\right)$ with coefficients $\left(c_{\mathbf{j}}\right)_{\mathbf{j}}$. Using the Cauchy-Schwarz inequality, we may write

$$
\begin{aligned}
\sum_{\mathbf{j} \in \mathscr{J}(m, n)}\left|c_{\mathbf{j}}\right| z_{\mathbf{j}}^{*} & \leq \sum_{j \geq 1} z_{j}^{*} \sum_{i_{1} \leq \cdots \leq i_{m-1} \leq j}\left|c_{(j, \mathbf{i})}\right| z_{i_{1}}^{*} \cdots z_{i_{m-1}}^{*} \\
& \leq \sum_{j \geq 1} z_{j}^{*}\left(\sum_{i_{1} \leq \cdots \leq i_{m-1} \leq j}\left|c_{(j, \mathbf{i})}\right|^{2}\right)^{1 / 2}\left(\sum_{i_{1} \leq \cdots \leq i_{m-1} \leq j} z_{i_{1}}^{* 2} \cdots z_{i_{m-1}}^{* 2}\right)^{1 / 2} .
\end{aligned}
$$

Now,

$$
\sum_{i_{1} \leq \cdots \leq i_{m-1} \leq j} z_{i_{1}}^{* 2} \cdots z_{i_{m-1}}^{* 2} \leq \sum_{i_{1} \leq \cdots \leq i_{m-1} \leq j} \frac{\|z\|^{2(m-1)}}{i_{1}^{\frac{m-1}{m}} \cdots i_{m-1}^{\frac{m-1}{m}}}
$$


For $k \leq m$ and $u \leq v$, we have

$$
\sum_{u \leq v} \frac{1}{u^{1-\frac{k}{m}}} \leq \int_{0}^{v} \frac{1}{u^{1-\frac{k}{m}}} d u=\frac{m}{k} v^{k / m} .
$$

By applying the above inequality for $k=1, \ldots, m-1$, an easy induction yields

$$
\begin{aligned}
\sum_{i_{1} \leq \cdots \leq i_{m-1} \leq j} z_{i_{1}}^{* 2} \cdots z_{i_{m-1}}^{* 2} & \leq \sum_{i_{m-1}=1}^{j} \sum_{i_{m-2}=1}^{i_{m-1}} \cdots \sum_{i_{1}=1}^{i_{2}} \frac{\|z\|^{2(m-1)}}{i_{1}^{1-\frac{1}{m}} i_{2}^{1-\frac{1}{m}} \cdots i_{m-1}^{1-\frac{1}{m}}} \\
& \leq \sum_{i_{m-1}=1}^{j} \sum_{i_{m-2}=1}^{i_{m-1}} \cdots \sum_{i_{2}=1}^{i_{3}} \frac{\|z\|^{2(m-1)} m}{i_{2}^{1-\frac{2}{m}} i_{3}^{1-\frac{1}{m}} \cdots i_{m-1}^{1-\frac{1}{m}}} \\
& \leq \cdots \\
& \leq\|z\|^{2(m-1)} \frac{m^{m-1}}{(m-1) !} j^{\frac{m-1}{m}} \\
& \leq e^{m-1}\|z\|^{2(m-1)} j^{\frac{m-1}{m}} .
\end{aligned}
$$

We then deduce that

$$
\sum_{\mathbf{j} \in \mathscr{J}(m, n)}\left|c_{\mathbf{j}}\right| z_{\mathbf{j}}^{*} \leq e^{\frac{m-1}{2}}\|z\|^{m} \sum_{j \geq 1}\left(\sum_{i_{1} \leq \cdots \leq i_{m-1} \leq j}\left|c_{(j, \mathbf{i})}\right|^{2}\right)^{1 / 2} \leq C^{m}\|z\|^{m}\|P\|
$$

where the conclusion comes from Lemma 2.5 with $p=1$. This shows that $z^{*} \in$ mon $\mathscr{P}\left({ }^{m} \ell_{\infty}\right)$, and hence the conclusion follows by the general properties of sets of monomial convergence (given at the beginning of this section).

\subsection{Proof of Theorem 2.2-lower inclusion}

The proof of Theorem 2.2-(a) is technically more demanding and needs further lemmas.

Lemma 2.6. Let $n \geq 1, p>1$ and $\rho>0$, and take $0<r_{i}<\rho$ for $i=1, \ldots, n$. Then for any sequence $\left(c_{\mathbf{i}}\right)_{\mathbf{i} \in \cup_{m \geq p} \mathscr{J}(m, n)}$ of nonnegative real numbers we have

$$
\begin{aligned}
\sum_{m=p}^{\infty} \sum_{\mathbf{i} \in \mathscr{J}(m, n)} c_{\mathbf{i}} r_{i_{1}} \ldots r_{i_{m}} \leq & {\left[\sum_{\mathbf{j} \in \mathscr{J}(p, n)}\left(r_{j_{1}} \cdots r_{j_{p}}\left(\prod_{l=1}^{j_{1}} \frac{1}{1-\left(\frac{r_{l}}{\rho}\right)^{2}}\right)^{1 / 2}\right)^{\frac{2 p}{p-1}}\right]^{\frac{p-1}{2 p}} } \\
& \times\left[\sum_{\mathbf{j} \in \mathscr{J}(p, n)}\left(\sum_{m \geq p} \sum_{\substack{\mathbf{i} \in \mathscr{L}(m-p, n) \\
i_{m-p} \leq j_{1}}} \rho^{2(m-p)} c_{(\mathbf{i}, \mathbf{j})}^{2}\right)^{\frac{1}{2} \times \frac{2 p}{p+1}}\right]^{\frac{p+1}{2 p}} .
\end{aligned}
$$


Proof. We begin by writing

$$
\begin{aligned}
\sum_{m=p}^{\infty} \sum_{\mathbf{i} \in \mathscr{J}(m, n)} c_{\mathbf{i}} r_{i_{1}} \ldots r_{i_{m}} & \\
& =\sum_{\mathbf{j} \in \mathscr{J}(p, n)} r_{j_{1}} \cdots r_{j_{p}} \sum_{m \geq p} \sum_{\substack{\mathbf{i} \in \mathscr{J}(m-p, n) \\
i_{m-p} \leq j_{1}}} \rho^{(m-p)} c_{(\mathbf{i}, \mathbf{j})} \rho^{-(m-p)} r_{i_{1}} \cdots r_{i_{m-p}} .
\end{aligned}
$$

We apply the Cauchy-Schwarz inequality (inside) to get:

$$
\begin{aligned}
& \sum_{m=p}^{\infty} \sum_{\mathbf{i} \in \mathscr{J}(m, n)} c_{\mathbf{i}} r_{i_{1}} \ldots r_{i_{m}} \leq \sum_{\mathbf{j} \in \mathscr{J}(p, n)} r_{j_{1}} \cdots r_{j_{p}}\left(\sum_{\substack { m \geq p \\
\begin{subarray}{c}{\mathbf{i} \in \mathscr{J}(m-p, n) \\
i_{m-p} \leq j_{1}{ m \geq p \\
\begin{subarray} { c } { \mathbf { i } \in \mathscr { J } ( m - p , n ) \\
i _ { m - p } \leq j _ { 1 } } }\end{subarray}} \rho^{2(m-p)} c_{(\mathbf{i}, \mathbf{j})}^{2}\right)^{1 / 2} \\
& \times\left(\sum_{\substack { m \geq p \\
\begin{subarray}{c}{\mathbf{i} \in \mathscr{L}(m-p, n) \\
i_{m-p} \leq j_{1}{ m \geq p \\
\begin{subarray} { c } { \mathbf { i } \in \mathscr { L } ( m - p , n ) \\
i _ { m - p } \leq j _ { 1 } } }\end{subarray}} r_{i_{1}}^{2} \cdots r_{i_{m-p}}^{2} \rho^{-2(m-p)}\right)^{1 / 2} \\
& \leq \sum_{\mathbf{j} \in \mathscr{J}(p, n)} r_{j_{1}} \cdots r_{j_{p}}\left(\prod_{l=1}^{j_{1}} \frac{1}{1-\left(\frac{r_{l}}{\rho}\right)^{2}}\right)^{1 / 2} \\
& \times\left(\sum_{\substack{m \geq p \mathbf{i} \in \mathscr{f}(m-p, n) \\
i_{m-p} \leq j_{1}}} \rho^{2(m-p)} c_{(\mathbf{i}, \mathbf{j})}^{2}\right)^{1 / 2} .
\end{aligned}
$$

We conclude by applying Hölder's inequality with the couple of conjugate exponents $\frac{2 p}{p+1}$ and $\frac{2 p}{p-1}$.

The strategy now will be to bound each factor in the preceding lemma. The first factor will be controlled by the condition given in Theorem 2.2 .

Lemma 2.7. Fix $p>1,0<\alpha<\rho$, and let $\left(r_{n}\right)_{n \in \mathbb{N}}$ be a nonincreasing sequence of nonnegative real numbers satisfying, for all $n \geq 1$,

$$
\left\{\begin{aligned}
r_{n} & <\rho \\
\frac{1}{\log (n+1)}\left(r_{1}^{2}+\cdots+r_{n}^{2}\right) & \leq \alpha^{2}
\end{aligned}\right.
$$

Then the sequence

$$
\left(\sum_{\mathbf{j} \in \mathcal{J}(p, n)}\left(r_{j_{1}} \cdots r_{j_{p}}\left(\prod_{l=1}^{j_{1}} \frac{1}{1-\left(\frac{r_{l}}{\rho}\right)^{2}}\right)^{1 / 2}\right)_{n}^{\frac{2 p}{p-1}}\right)_{n}
$$


is bounded.

Proof. It is enough to prove that

$$
H:=\sum_{j_{1}=1}^{\infty} r_{j_{1}}^{\frac{2 p}{p-1}}\left(\prod_{l=1}^{j_{1}} \frac{1}{1-\left(\frac{r_{l}}{\rho}\right)^{2}}\right)^{\frac{p}{p-1}} \sum_{j_{1} \leq j_{2} \leq \cdots \leq j_{p}}\left(r_{j_{2}} \cdots r_{j_{p}}\right)^{\frac{2 p}{p-1}}
$$

is finite. We first consider the last sum. Because $\left(r_{n}\right)_{n}$ is nonincreasing, it is plain that, for any $n \geq 1, r_{n} \leq \alpha \sqrt{\log (n+1)} / \sqrt{n}$. We will use that there is a constant $A_{p} \geq 1$ such for all $a \in \mathbb{N}$ we have

$$
\sum_{k \geq a} \frac{(\log (k+1))^{\frac{p}{p-1}}}{k^{\frac{p}{p-1}}} \leq A_{p} \frac{1+(\log a)^{\frac{p}{p-1}}}{a^{\frac{1}{p-1}}} .
$$

This implies for every $j_{1}$

$$
\begin{aligned}
\sum_{j_{1} \leq j_{2} \leq \cdots \leq j_{p}} & \left(r_{j_{2}} \cdots r_{j_{p}}\right)^{\frac{2 p}{p-1}} \leq \sum_{j_{2}, \cdots, j_{p} \geq j_{1}}\left(r_{j_{2}} \cdots r_{j_{p}}\right)^{\frac{2 p}{p-1}} \\
& \ll\left(\sum_{k=j_{1}}^{\infty} \frac{(\log (k+1))^{\frac{p}{p-1}}}{k^{\frac{p}{p-1}}}\right)^{p-1} \ll \frac{\left(1+\left(\log j_{1}\right)^{\frac{p}{p-1}}\right)^{p-1}}{j_{1}} \ll \frac{1+\left(\log j_{1}\right)^{p}}{j_{1}},
\end{aligned}
$$

where the constant in the last inequality only depends on $\alpha$ and $p$. Furthermore,

$$
\prod_{l=1}^{j_{1}} \frac{1}{1-\left(\frac{r_{l}}{\rho}\right)^{2}}=\exp \left(-\sum_{l=1}^{j_{1}} \log \left(1-\frac{r_{l}^{2}}{\rho^{2}}\right)\right) .
$$

Let $\varepsilon>0$ be such that $\alpha^{2}(1+\varepsilon)<\rho^{2}$. Since $\left(r_{n}\right)_{n}$ goes to zero, there exists some $A>0$ such that

$$
-\sum_{l=1}^{j_{1}} \log \left(1-\frac{r_{l}^{2}}{\rho^{2}}\right) \leq A+(1+\varepsilon) \sum_{l=1}^{j_{1}} \frac{r_{l}^{2}}{\rho^{2}} \leq A+(1+\varepsilon) \frac{\alpha^{2}}{\rho^{2}} \log j_{1}
$$

for any $j_{1} \geq 1$ (use again that $\lim _{x \rightarrow 0} \frac{-\log (1-x)}{x}=1$ ). This yields

$$
\prod_{l=1}^{j_{1}} \frac{1}{1-\left(\frac{r_{l}}{\rho}\right)^{2}} \ll j_{1}^{\delta}
$$

for some $\delta<1$. Hence,

$$
H \ll \sum_{j_{1} \geq 1} \frac{\left(\log \left(j_{1}+1\right)\right)^{\frac{p}{p-1}}\left(1+\log j_{1}\right)^{p}}{\left(j_{1}\right)^{1+(1-\delta) \frac{p}{p-1}}} .
$$

The last sum is convergent and this completes the proof. 
Finally, we are ready to give the

Proof of Theorem 2.2-(a). Take $z \in B_{\ell_{\infty}}$ such that

$$
A:=\limsup _{n \rightarrow \infty} \frac{1}{\log n} \sum_{j=1}^{n} z_{j}^{* 2}<1 .
$$

We write for simplicity $r_{n}$ for $z_{n}^{*}$, and we are going to show that $r \in \operatorname{mon} H_{\infty}\left(B_{\ell_{\infty}}\right)$ (see the preliminaries of this section). Choose $A<\alpha<\rho<1$. Moreover, we know that changing a finite number of terms does not change the property $r \in \operatorname{mon} H_{\infty}\left(B_{\ell_{\infty}}\right)$ (see again the preliminaries), hence we may assume that for all $n \geq 1$

$$
\left\{\begin{aligned}
r_{n} & <\rho \\
\frac{1}{\log (n+1)}\left(r_{1}^{2}+\cdots+r_{n}^{2}\right) & \leq \alpha^{2}
\end{aligned}\right.
$$

Now we choose $p>1$ and $\kappa>1$ such that $\kappa \rho\left(1+\frac{1}{p}\right)<1$, and consider for each fixed $f \in H_{\infty}\left(B_{\ell_{\infty}}\right)$ and for each $n$ the decomposition

$$
\sum_{\alpha \in \mathbb{N}^{n}}\left|c_{\alpha}\right| r^{\alpha}=\sum_{m=1}^{p-1} \sum_{\mathbf{j} \in \mathscr{J}(m, n)}\left|c_{\mathbf{j}}\right| r_{j_{1}} \ldots r_{j_{m}}+\sum_{m=p}^{\infty} \sum_{\mathbf{j} \in \mathscr{J}(m, n)}\left|c_{\mathbf{j}}\right| r_{j_{1}} \ldots r_{j_{m}} .
$$

Since

$$
r \in \ell_{\frac{2 k}{k-1}, \infty} \text { for all } k,
$$

we deduce from Theorem 2.1 (here in fact only the weaker version from (20) is needed) that the first summand is bounded by a constant independent of $n$. Moreover, by Lemmas 2.6 and 2.7, the second summand can be majorized as follows:

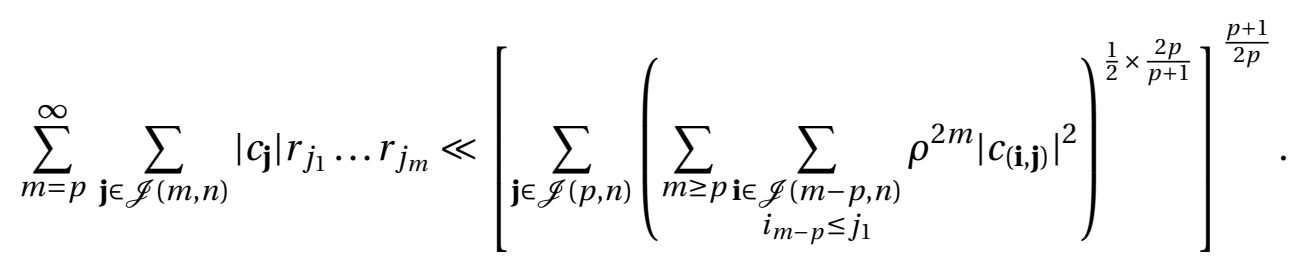

Now observe that we have $\left\|P_{m}\right\| \leq\|f\|$ for all $m$, where $P_{m}$ denotes the unique $m$-homogeneous polynomial from the Taylor series expansion $f=\sum_{m \geq 0} P_{m}$. Hence we obtain from the fact that the $\ell_{1}$-norm dominates the $\ell_{2}$-norm, Min- 
kowki's inequality and Lemma 2.5 that

$$
\begin{aligned}
\sum_{m=p}^{\infty} \sum_{\mathbf{j} \in \mathscr{J}(m, n)}\left|c_{\mathbf{j}}\right| r_{j_{1}} \ldots r_{j_{m}} \ll & {\left[\sum_{\mathbf{j} \in \mathcal{J}(p, n)}\left(\sum_{m \geq p}\left(\sum_{\substack{\mathbf{i} \in \mathscr{J}(m-p, n) \\
i_{m-p} \leq j_{1}}} \rho^{2 m}\left|c_{(\mathbf{i}, \mathbf{j})}\right|^{2}\right)^{\frac{1}{2}}\right]^{\frac{2 p}{p+1}}\right]^{\frac{p+1}{2 p}} } \\
& \leq \sum_{m \geq p} \rho^{m}\left[\sum_{\mathbf{j} \in \mathscr{J}(p, n)}\left(\sum_{\mathbf{i} \in \mathscr{J}(m-p, n) i_{m-p} \leq j_{1}}\left|c_{(\mathbf{i}, \mathbf{j})}\right|^{2}\right)^{\frac{1}{2} \times \frac{2 p}{p+1}}\right]^{\frac{p+1}{2 p}} \\
& \leq \sum_{m \geq p}\left[\rho\left(1+\frac{1}{p}\right) \kappa\right]^{m}\|f\| .
\end{aligned}
$$

This completes the proof.

\subsection{The probabilistic device}

The upper inclusions in Theorem 2.1 and Theorem 2.2 are based on the following probabilistic device known as the Kahane-Salem-Zygmund inequality (see e.g. [22, Chapter 6, Theorem 4]): There is a universal constant $C_{\mathrm{KSZ}}>0$ such that for any $m, n$ and any family $\left(a_{\alpha}\right)_{\alpha \in \Lambda(m, n)}$ of complex numbers there exists a choice of signs $\varepsilon_{\alpha}= \pm 1$ for which

$$
\sup _{z \in \mathbb{D}^{n}}\left|\sum_{\alpha \in \Lambda(m, n)} \varepsilon_{\alpha} a_{\alpha} z^{\alpha}\right| \leq C_{\mathrm{KSZ}} \sqrt{n \log m \sum_{\alpha}\left|a_{\alpha}\right|^{2}} .
$$

Let us start with the proof of the upper inclusion of Theorem 2.1. As we have already mentioned earlier (see [22), this result is from [14], where it appears as a special case of a more general result proved through more sophisticated probabilistic argument. For the sake of completeness we here prefer to give a direct argument based on (27).

Proof of the upper inclusion in Theorem 2.1. Take $z \in \operatorname{mon} \mathscr{P}\left({ }^{m} \ell_{\infty}\right)$. We show that the decreasing rearrangement $r=z^{*} \in \ell_{\frac{2 m}{m-1}, \infty}$. Since $r \in \operatorname{mon} \mathscr{P}\left({ }^{m} \ell_{\infty}\right)$, a straightforward closed graph argument (see also [14, Lemma 4.1]) shows that there is a constant $C(z)>0$ such that for every $Q \in \mathscr{P}\left({ }^{m} \ell_{\infty}\right)$ we have

$$
\sum_{\alpha \in \mathbb{N}_{0}^{(\mathbb{N})}}\left|c_{\alpha}(Q) r^{\alpha}\right| \leq C(z)\|Q\|
$$


By (27) for each $n$ there are signs $\varepsilon_{\alpha}= \pm 1, \alpha \in \Lambda(m, n)$ such that the $m$-homogeneous polynomial

$$
P(u)=\sum_{\alpha \in \Lambda(m, n)} \varepsilon_{\alpha} \frac{m !}{\alpha !} u^{\alpha}, u \in \mathbb{C}^{n}
$$

satisfies

$$
\|P\| \leq C_{\mathrm{KSZ}} \sqrt{n \log m \sum_{\alpha \in \Lambda(m, n)}\left|c_{\alpha}(P)\right|^{2}} .
$$

But by the multinomial formula we have

$$
\sum_{\alpha \in \Lambda(m, n)}\left|c_{\alpha}(P)\right|^{2}=\sum_{\alpha \in \Lambda(m, n)}\left(\frac{m !}{\alpha !}\right)^{2} \leq m ! \sum_{\alpha \in \Lambda(m, n)} \frac{m !}{\alpha !}=m ! n^{m},
$$

and hence we conclude from (28) and (29) (and another application of the multinomial formula) that

$$
\left(\sum_{j=1}^{n} r_{j}\right)^{m}=\sum_{\alpha \in \Lambda(m, n)} \frac{m !}{\alpha !} r^{\alpha} \leq C(z) C_{\mathrm{KSZ}} \sqrt{m ! \log m} n^{\frac{m+1}{2}}
$$

Finally, this shows that for all $n$ we have

$$
r_{n} \leq \frac{1}{n} \sum_{j=1}^{n} r_{j} \leq C(z) C_{\mathrm{KSZ}}(m ! \log m)^{\frac{1}{m}} n^{\frac{m+1}{2 m}-1} \ll \frac{1}{n^{\frac{m-1}{2 m}}},
$$

the conclusion.

A similar argument leads to the

Proof of the upper inclusion in Theorem 2.2. Let us fix some $z \in \operatorname{mon} H_{\infty}\left(B_{\ell_{\infty}}\right)$. Then $z \in B_{c_{0}}$ and without loss of generality we may assume that $r=z$ is nonincreasing. Again a closed graph argument assures that there is $C(z)>0$ such that for every $f \in H_{\infty}\left(B_{\ell_{\infty}}\right)$

$$
\sum_{\alpha}\left|c_{\alpha}(f)\right| r^{\alpha} \leq C(z)\|f\|
$$

For each $m, n$ and $a_{\alpha}=r^{\alpha}, \alpha \in \Lambda(m, n)$ we choose signs $\varepsilon_{\alpha}$ according to 27), and define $f(u)=\sum_{\alpha \in \Lambda(m, n)} \varepsilon_{\alpha} r^{\alpha} u^{\alpha}, u \in \mathbb{D}^{n}$. Then the preceding estimate gives

$$
\begin{aligned}
\sum_{\alpha \in \Lambda(m, n)} r^{2 \alpha} & =\sum_{\alpha \in \Lambda(m, n)}\left|\varepsilon_{\alpha} r^{\alpha}\right| r^{\alpha} \leq C(z)\|f\| \\
& \leq C(z) C_{\mathrm{KSZ}}\left(n \log m \sum_{\alpha \in \Lambda(m, n)}\left|r^{\alpha}\right|^{2}\right)^{\frac{1}{2}}=A \sqrt{n \log m}\left(\sum_{\alpha \in \Lambda(m, n)} r^{2 \alpha}\right)^{\frac{1}{2}} .
\end{aligned}
$$


This implies

$$
\left(\sum_{\alpha \in \Lambda(m, n)} r^{2 \alpha}\right)^{\frac{1}{2}} \leq A \sqrt{n \log m} .
$$

Now,

$$
\left(r_{1}^{2}+\cdots+r_{n}^{2}\right)^{m} \leq m ! \sum_{\alpha \in \Lambda(m, n)} r^{2 \alpha} .
$$

Using Stirling's formula and taking the power $1 / m$, we get

$$
r_{1}^{2}+\cdots+r_{n}^{2} \leq A^{\frac{1}{m}} m e^{-1} m^{\frac{1}{2 m}} n^{\frac{1}{m}}(\log m)^{\frac{1}{m}} .
$$

We then choose $m=\lfloor\log n\rfloor$ so that $e^{-1} n^{1 / m} \rightarrow 1$. This yields

$$
r_{1}^{2}+\cdots+r_{n}^{2} \leq \log n \times \exp \left(\left(\frac{1}{2}+o(1)\right) \frac{\log \log n}{\log n}\right),
$$

and we immediately deduce

$$
\limsup _{n \rightarrow \infty} \frac{1}{\log n} \sum_{j=1}^{n} r_{j}^{2} \leq 1
$$

Moreover, the converse is false, since if we consider a decreasing sequence $\left(r_{n}\right)$ satisfying, for large values of $n$,

$$
r_{1}^{2}+\cdots+r_{n}^{2}=\log n \times \exp \left(\frac{\log \log n}{\log n}\right)
$$

then $\limsup _{n \rightarrow \infty} \frac{1}{\log n} \sum_{j=1}^{n} r_{j}^{2} \leq 1$ whereas $\left(r_{n}\right) \notin \operatorname{mon} H_{\infty}\left(B_{\ell_{\infty}}\right)$ since this would contradict 30 .

Remark 2.8. The same argument gives also informations on the constant $C$ appearing in (23). More precisely, if there exists $A, C>0$ such that, for every $z \in \ell_{\frac{2 m}{m-1}, \infty}$ and for every $P \in \mathscr{P}\left({ }^{m} \ell_{\infty}\right)$, we have

$$
\sum_{|\alpha|=m}\left|c_{\alpha}(P) z^{\alpha}\right| \leq A C^{m}\|z\|^{m}\|P\|,
$$

then we claim that $C \geq e^{1 / 2}$. Indeed, provided (31) is satisfied, and arguing as in the proof of Theorem 2.2, we see that for any $0<r_{1}, \ldots, r_{n}$,

$$
\left(r_{1}^{2}+\cdots+r_{n}^{2}\right)^{m / 2} \leq A C^{m}\|r\|^{m} C_{\mathrm{KSZ}} \sqrt{m !} \sqrt{n \log m} .
$$


We choose $r_{j}=\frac{1}{j \frac{m-1}{2 m}}$ so that $\|r\|=1$ and

$$
r_{1}^{2}+\cdots+r_{n}^{2}=\sum_{j=1}^{n} \frac{1}{j^{1-\frac{1}{m}}} \geq \int_{1}^{n} \frac{d x}{x^{1-\frac{1}{m}}} \geq m n^{\frac{1}{m}}-m .
$$

Hence,

$$
C \geq \frac{1}{\left(A C_{\mathrm{KSZ}}\right)^{\frac{1}{m}}} \times \frac{1}{(\log m)^{\frac{1}{2 m}}} \times \frac{1}{(m !)^{\frac{1}{2 m}}} \times\left(m-\frac{m}{n^{\frac{1}{m}}}\right)^{\frac{1}{2}} .
$$

Letting $n$ to infinity and then $m$ to infinity, and using

$$
\lim _{m \rightarrow+\infty} \frac{m}{(m !)^{\frac{1}{m}}}=e
$$

we get the claim. In fact, an analysis of the proof of Theorem 2.1-lower inclusion shows that (31) is satisfied with $C$ any constant greater than $(2 e)^{1 / 2}$.

\subsection{Dismissing candidates}

A natural question seems to be whether or not there is a sequence space $X$ (i.e., a vector space $X$ of complex sequences) such that $X \cap B_{\ell_{\infty}}=\operatorname{mon} H_{\infty}\left(B_{\ell_{\infty}}\right)$. The first natural candidate to do that job was $\ell_{2}$ (see again (17), (20), and (21)). But, as we already have seen in (13), the sequence $\left(p_{n}^{-1 / 2}\right)_{n}$ belongs to mon $H_{\infty}\left(B_{\ell_{\infty}}\right)$ although it is not in $\ell_{2}$. The three other natural candidates are the spaces $\ell_{2,0}$, $\ell_{2, \infty}$ and $\ell_{2, \log }$ :

$$
\begin{aligned}
& \ell_{2,0}=\left\{z \in \ell_{\infty} \mid \lim _{n} z_{n}^{*} n^{1 / 2}=0\right\} \\
& \ell_{2, \infty}=\left\{z \in \ell_{\infty} \mid \exists c \forall n: z_{n}^{*} \leq c \frac{1}{\sqrt{n}}\right\} \\
& \ell_{2, \log }=\left\{z \in \ell_{\infty} \mid \exists c \forall n: z_{n}^{*} \leq c \sqrt{\frac{\log n}{n}}\right\} .
\end{aligned}
$$

Theorem 2.2 shows that neither $\ell_{2,0}$ nor $\ell_{2, \log }$ are the proper spaces since we have

$$
\ell_{2,0} \cap B_{\ell_{\infty}} \varsubsetneqq \mathbf{B} \subset \operatorname{mon} H_{\infty}\left(B_{\ell_{\infty}}\right) \subset \overline{\mathbf{B}} \varsubsetneqq \ell_{2, \log } \cap B_{\ell_{\infty}}
$$

(recall the definition of $\overline{\mathbf{B}}$ from (11)). We prove this: Note first that

$$
\left(\frac{c}{\sqrt{n}}\right)_{n \in \mathbb{N}} \begin{cases}\in \operatorname{mon} H_{\infty}\left(B_{\ell_{\infty}}\right) & \text { for } c<1 \\ \notin \operatorname{mon} H_{\infty}\left(B_{\ell_{\infty}}\right) & \text { for } 1<c\end{cases}
$$

since

$$
\limsup _{n \rightarrow \infty} \frac{1}{\log n} \sum_{j=1}^{n} \frac{1}{j}=1
$$


Now, (26) and (33) immediately give $\ell_{2,0} \cap B_{\ell_{\infty}} \varsubsetneqq \mathbf{B}$. The last inclusion in (32) follows from the fact that $\limsup _{n} \frac{1}{\log n} \sum_{1}^{n} z_{j}^{* 2}<\infty$ obviously implies that $z \in$ $\ell_{2, \log .}$ On the other hand,

$$
\limsup _{n \rightarrow \infty} \frac{1}{\log n} \sum_{j=1}^{n}\left(\sqrt{\frac{\log j}{j}}\right)^{2} \geq \limsup _{n \rightarrow \infty} \frac{1}{\log n} \sum_{j=1}^{n} \frac{\log 3}{j}=\log 3>1
$$

gives $\left(\sqrt{\frac{\log n}{n}}\right)_{n} \notin \overline{\mathbf{B}}$ and shows that this inclusion is also strict.

In view of (33) the following interesting problem remains open:

$$
\left(\frac{1}{\sqrt{n}}\right)_{n} \in \operatorname{mon} H_{\infty}\left(B_{\ell_{\infty}}\right) ?
$$

In fact, Theorem 2.2, even proves that there is no sequence space $X$ at all for which mon $H_{\infty}\left(B_{\ell_{\infty}}\right)=X \cap B_{\ell_{\infty}}$ : Indeed, assume that such an $X$ exists. By (33) we have that $\left(\frac{1}{2} n^{-1 / 2}\right)_{n \geq 9} \in$ mon $H_{\infty}\left(B_{\ell_{\infty}}\right)$, and therefore by assumption $\left(\frac{3}{2} n^{-1 / 2}\right)_{n \geq 9} \in X \cap B_{\ell_{\infty}}$. But this implies $\left(\frac{3}{2} n^{-1 / 2}\right)_{n \geq 9} \in \operatorname{mon} H_{\infty}\left(B_{\ell_{\infty}}\right)$, a contradiction to (33).

Finally, we compare $\ell_{2, \infty} \cap B_{\ell_{\infty}}$ with mon $H_{\infty}\left(B_{\ell_{\infty}}\right)$. Again by (33) we see that $B_{\ell_{2, \infty}} \subset \operatorname{mon} H_{\infty}\left(B_{\ell_{\infty}}\right)$, and moreover that there are sequences in $\ell_{2, \infty} \cap B_{\ell_{\infty}}$ that do not belong to mon $H_{\infty}\left(B_{\ell_{\infty}}\right)$. But it also can be shown that

$$
\operatorname{mon} H_{\infty}\left(B_{\ell_{\infty}}\right) \nsubseteq \ell_{2, \infty}
$$

the proof is now slightly more complicated: Take a strictly increasing sequence of non-negative integers $\left(n_{k}\right)_{k}$ with $n_{1}>1$, satisfying that the sequence $\left(\frac{k+1}{n_{k}}\right)_{k}$ is strictly decreasing and

$$
\sum_{k=1}^{\infty} \frac{k+1}{n_{k}}<1
$$

(take for example $n_{k}=a^{k^{2}(k+1)}$ for $a \in \mathbb{N}$ big enough). Now we define

$$
r_{j}= \begin{cases}\sqrt{\frac{1}{n_{1}}} & 1 \leq j \leq n_{1} \\ \sqrt{\frac{k+1}{n_{k+1}}} & n_{k}<j \leq n_{k+1}, \quad k=1,2, \ldots\end{cases}
$$

The sequence $\left(r_{n}\right)$ is decreasing to 0 . Clearly, $n_{k} r_{n_{k}}^{2}=k$ for all $k$. Thus $\left(r_{n}\right)$ does not belong to $\ell_{2, \infty}$. But for $n>n_{1}$, if $n_{k}<n \leq n_{k+1}$ and $\lim _{k} \frac{k+1}{\log n_{k}}=0$ (a 
condition satisfied by the above example), then

$$
\begin{aligned}
\frac{1}{\log n} \sum_{j=1}^{n} r_{j}^{2}= & \frac{1}{\log n}\left(\sum_{j=1}^{n_{1}} \frac{1}{n_{1}}+\sum_{h=1}^{k-1} \sum_{j=n_{h}+1}^{n_{h+1}} r_{j}^{2}+\sum_{j=n_{k}+1}^{n} r_{j}^{2}\right) \\
\leq & \frac{1}{\log n}\left(1+\sum_{h=1}^{k-1} \frac{n_{h+1}-n_{h}}{n_{h+1}}(h+1)+\frac{n_{k+1}-n_{k}}{n_{k+1}}(k+1)\right) \\
& \leq \frac{1}{\log n_{1}}+\sum_{h=1}^{k-1} \frac{h+1}{\log n_{h+1}}+\frac{k+1}{\log n_{k}}<\sum_{h=1}^{\infty} \frac{h+1}{n_{h}}+\frac{k+1}{\log n_{k}} .
\end{aligned}
$$

Hence $\limsup _{n \rightarrow \infty} \frac{1}{\log n} \sum_{j=1}^{n} r_{j}^{2}<1$, and therefore $\left(r_{n}\right)_{n} \in \operatorname{mon} H_{\infty}\left(B_{\ell_{\infty}}\right)$.

\section{Series expansion of $H_{p}$-functions in infinitely many variables}

We draw now our attention to functions on $\mathbb{T}^{\infty}$, the infinite dimensional polytorus. We recall that $m$ denotes the product of the normalized Lebesgue measure on $\mathbb{T}^{\infty}$. Given a function $f \in L_{p}\left(\mathbb{T}^{\infty}\right)$, its Fourier coefficients $(\hat{f}(\alpha))_{\left.\alpha \in \mathbb{Z}^{(\mathbb{N}}\right)}$ are defined by $\hat{f}(\alpha)=\int_{\mathbb{T} \infty} f(w) w^{-\alpha} d m(w)=\left\langle f, w^{\alpha}\right\rangle$ where $w^{\alpha}=w_{1}^{\alpha_{1}} \ldots w_{n}^{\alpha_{n}}$ if $\alpha=\left(\alpha_{1} \ldots \alpha_{n}, 0, \ldots\right)$ for $w \in \mathbb{T}^{\infty}$, and the bracket $\langle\cdot, \cdot\rangle$ refers to the duality between $L_{p}\left(\mathbb{T}^{\infty}\right)$ and $L_{q}\left(\mathbb{T}^{\infty}\right)$ for $1 / p+1 / q=1$. With this, for $1 \leq p \leq \infty$ the Hardy spaces are defined as

$$
H_{p}\left(\mathbb{T}^{\infty}\right)=\left\{f \in L_{p}\left(\mathbb{T}^{\infty}\right) \mid \hat{f}(\alpha)=0, \forall \alpha \in \mathbb{Z}^{(\mathbb{N})} \backslash \mathbb{N}_{0}^{(\mathbb{N})}\right\}
$$

We will also consider, for each $m$, the following closed subspace

$$
H_{p}^{m}\left(\mathbb{T}^{\infty}\right)=\left\{f \in H_{p}\left(\mathbb{T}^{\infty}\right)|\hat{f}(\alpha) \neq 0 \Rightarrow| \alpha \mid=m\right\}
$$

of $L_{p}\left(\mathbb{T}^{\infty}\right)$. By [8, Section 9] this is the completion of the $m$-homogeneous trigonometric polynomials (functions on $\mathbb{T}^{\infty}$ that are finite sums of the form $\left.\sum_{|\alpha|=m} c_{\alpha} w^{\alpha}\right)$. It is important to note that

$$
H_{q}^{m}\left(\mathbb{T}^{\infty}\right)=H_{p}^{m}\left(\mathbb{T}^{\infty}\right), 1 \leq p, q<\infty \text { and } m \in \mathbb{N}
$$

this was first observed in by [8, 9.1 Theorem] (here it also follows from Lemma 2.3 and a density argument). 
In analogy to (14) and (15) we define for every for $1 \leq p \leq \infty$ and $m \in \mathbb{N}$ the following two sets of monomial convergence:

$$
\begin{aligned}
& \operatorname{mon} H_{p}\left(\mathbb{T}^{\infty}\right)=\left\{z \in \mathbb{C}^{\mathbb{N}}\left|\sum_{\alpha}\right| \hat{f}(\alpha) z^{\alpha} \mid<\infty \text { for all } f \in H_{p}\left(\mathbb{T}^{\infty}\right)\right\} \\
& \operatorname{mon} H_{p}^{m}\left(\mathbb{T}^{\infty}\right)=\left\{z \in \mathbb{C}^{\mathbb{N}}\left|\sum_{\alpha}\right| \hat{f}(\alpha) z^{\alpha} \mid<\infty \text { for all } f \in H_{p}^{m}\left(\mathbb{T}^{\infty}\right)\right\} .
\end{aligned}
$$

Obviously both sets are increasing in $p$. In sections 3.1 and 3.2 we will prove that

$$
\begin{aligned}
& \operatorname{mon} H_{\infty}\left(\mathbb{T}^{\infty}\right)=\operatorname{mon} H_{\infty}\left(B_{c_{0}}\right)=\operatorname{mon} H_{\infty}\left(B_{\ell_{\infty}}\right) \\
& \operatorname{mon} H_{\infty}^{m}\left(\mathbb{T}^{\infty}\right)=\operatorname{mon} \mathscr{P}\left({ }^{m} c_{0}\right)=\operatorname{mon} \mathscr{P}\left({ }^{m} \ell_{\infty}\right),
\end{aligned}
$$

which by Theorem 2.1 and Theorem 2.2 then in particular implies that

$$
\operatorname{mon} H_{p}\left(\mathbb{T}^{\infty}\right) \subset \overline{\mathbf{B}} \text { and } \operatorname{mon} H_{p}^{m}\left(\mathbb{T}^{\infty}\right) \subset \ell_{\frac{m-1}{2 m}, \infty}
$$

But we are going to see in this section that a much more precise description is possible.

\subsection{The homogeneous case}

The homogeneous case can be solved completely.

\section{Theorem 3.1.}

$$
\operatorname{mon} H_{p}^{m}\left(\mathbb{T}^{\infty}\right)= \begin{cases}\ell_{2} & \text { for } 1 \leq p<\infty \\ \ell_{\frac{2 m}{m-1}, \infty} & \text { for } p=\infty .\end{cases}
$$

Moreover, there is $C>0$ such that if $z \in \operatorname{mon} H_{p}^{m}\left(\mathbb{T}^{\infty}\right)$ and $f \in H_{p}^{m}\left(\mathbb{T}^{\infty}\right)$, then

$$
\sum_{|\alpha|=m}\left|\hat{f}(\alpha) z^{\alpha}\right| \leq C^{m}\|z\|^{m}\|f\|_{p}
$$

where $\|z\|$ is the norm in the corresponding sequence space (here $1 \leq C \leq \sqrt{2}$ for $1 \leq p \leq 2$ and $C=1$ for $2 \leq p<\infty$ ).

Again we prepare the proof with some lemmas of independent interest. We deal with two separate situations: $p=\infty$ and $p=2$ (covering the case for arbitrary $1 \leq p<\infty$ ). The first case will follow from Theorem 2.1, after showing that $H_{\infty}^{m}\left(\mathbb{T}^{\infty}\right)$ can be identified with $\mathscr{P}\left({ }^{m} c_{0}\right)$. The basic idea here is, given a polynomial on $c_{0}$, extend it to $\ell_{\infty}$ and then restrict it to $\mathbb{T}^{\infty}$. Let us very briefly recall how $m$-homogeneous polynomials on a Banach space $X$ can be extended to its bidual (see [16, Section 6] or [15, Proposition 1.53]). First of all, every $m$-linear mapping $A: X \times \cdots \times X \rightarrow \mathbb{C}$ has a unique extension (called the Arens extension) 
$\tilde{A}: X^{* *} \times \cdots \times X^{* *} \rightarrow \mathbb{C}$ such that for all $j=1, \ldots, n$, all $x_{k} \in X$ and $z_{k} \in X^{* *}$, the mapping that to $z \in X^{* *}$ associates $\tilde{A}\left(x_{1}, \ldots, x_{j-1}, z, z_{j+1}, \ldots, z_{m}\right)$ is weak*continuous. Now, given $P \in \mathscr{P}\left({ }^{m} X\right)$, we take its associated symmetric $m$-linear form $A$ and define its Aron-Berner extension $\tilde{P} \in \mathscr{P}\left({ }^{m} X^{* *}\right)$ by $\tilde{P}(z)=\tilde{A}(z, \ldots, z)$. By [9, Theorem 3] we have

$$
\sup _{x \in B_{X}}|P(x)|=\sup _{z \in B_{X^{* *}}}|\tilde{P}(z)|
$$

Hence, the operator

$$
A B: \mathscr{P}\left({ }^{m} X\right) \rightarrow \mathscr{P}\left({ }^{m} X^{* *}\right), A B(P)=\tilde{P}
$$

is a linear isometry.

\section{Lemma 3.2. The mapping}

$$
\psi: \mathscr{P}\left({ }^{m} c_{0}\right) \rightarrow H_{\infty}^{m}\left(\mathbb{T}^{\infty}\right), \psi(P)(w)=A B(P)(w)
$$

is a surjective isometry.

Proof. Let us note first that, by the very definition of the Aron-Berner extension, for each $\alpha \in \mathbb{N}_{0}^{(\mathbb{N})}$, the monomial $x \in c_{0} \mapsto x^{\alpha}$ is extended to the monomial $z \in \ell_{\infty} \mapsto z^{\alpha}$. Then the set of finite sums of the type $\sum_{|\alpha|=m} c_{\alpha} x^{\alpha}$ is bijectively and isometrically mapped onto the set of $m$-homogeneous trigonometric polynomials. By [15, Propositions 1.59 and 2.8] the monomials on $c_{0}$ with $|\alpha|=m$ generate a dense subspace of $\mathscr{P}\left({ }^{m} c_{0}\right)$. On the other hand, by [8, Section 9] the trigonometric polynomials are dense in $H_{\infty}^{m}\left(\mathbb{T}^{\infty}\right)$. This gives the result.

To deal with the case $1 \leq p<\infty$ we need the following lemma.

Lemma 3.3. For $1 \leq p<\infty$ and $m \in \mathbb{N}$

$$
\operatorname{mon} H_{p}^{m}\left(\mathbb{T}^{\infty}\right) \subset \operatorname{mon} H_{p}^{m-1}\left(\mathbb{T}^{\infty}\right) \text {. }
$$

Proof. Let $0 \neq z \in$ mon $H_{p}^{m}\left(\mathbb{T}^{\infty}\right)$ and $f \in \operatorname{mon} H_{p}^{m-1}\left(\mathbb{T}^{\infty}\right)$. We choose $z_{i_{0}} \neq 0$ and define $\tilde{f}(w)=w_{i_{0}} f(w)$. Let us see that $\tilde{f} \in H_{p}^{m}\left(\mathbb{T}^{\infty}\right)$; indeed, take a sequence $\left(f_{n}\right)_{n}$ of $(m-1)$-homogeneous trigonometric polynomials that converges in the space $L_{p}\left(\mathbb{T}^{\infty}\right)$ to $f$. Each $f_{n}$ is a finite sum of the type $\sum_{|\alpha|=m-1} c_{\alpha}\left(f_{n}\right) w^{\alpha}$. We define for $w \in \mathbb{T}^{\infty}$

$$
\tilde{f}_{n}(w)=w_{i_{0}} f_{n}(w)=\sum_{|\alpha|=m-1} c_{\alpha}\left(f_{n}\right) w_{1}^{\alpha_{1}} \cdots w_{i_{0}}^{\alpha_{i_{0}}+1} \cdots w_{k}^{\alpha_{k}}
$$


Clearly, $\tilde{f}_{n}$ is an $m$-homogeneous trigonometric polynomial. Moreover

$$
\begin{aligned}
\left(\int_{\mathbb{T} \infty}\left|w_{i_{0}} f_{n}(w)-w_{i_{0}} f(w)\right|^{p} d m(w)\right)^{\frac{1}{p}}= & \left(\int_{\mathbb{T}^{\infty}}\left|w_{i_{0}}\right|^{p}\left|f_{n}(w)-f(w)\right|^{p} d m(w)\right)^{\frac{1}{p}} \\
& \leq\left(\int_{\mathbb{T}^{\infty}}\left|f_{n}(w)-f(w)\right|^{p} d m(w)\right)^{\frac{1}{p}}
\end{aligned}
$$

The last term converges to 0 , hence $\left(\tilde{f}_{n}\right)_{n}$ converges in $L_{p}\left(\mathbb{T}^{\infty}\right)$ to $\tilde{f}$ and $\tilde{f} \in$ $H_{p}^{m}\left(\mathbb{T}^{\infty}\right)$. We compute now the Fourier coefficients:

$$
\begin{aligned}
& \hat{\tilde{f}}(\alpha)=\int_{\mathbb{T} \infty} \tilde{f}(w) w^{-\alpha} d m(w)=\int_{\mathbb{T} \infty} w_{i_{0}} f(w) w^{-\alpha} d m(w) \\
&=\int_{\mathbb{T} \infty} f(w) w_{1}^{-\alpha_{1}} \cdots w_{i_{0}}^{-\alpha_{i_{0}}+1} \cdots w_{k}^{-\alpha_{k}} d m(w) \\
&=\int_{\mathbb{T} \infty} f(w) w_{1}^{-\alpha_{1}} \cdots w_{i_{0}}^{-\left(\alpha_{i_{0}}-1\right)} \cdots w_{k}^{-\alpha_{k}} d m(w) \\
& \quad=\hat{f}\left(\alpha_{1}, \ldots, \alpha_{i_{0}}-1, \ldots, \alpha_{k}\right) .
\end{aligned}
$$

That is

$$
\hat{\tilde{f}}(\alpha)= \begin{cases}\hat{f}(\beta) & \text { if } \alpha=\left(\beta_{1}, \ldots, \beta_{i_{0}}+1, \ldots, \beta_{k}\right) \\ 0 & \text { otherwise }\end{cases}
$$

and this gives

$$
\begin{aligned}
\sum_{\beta}\left|\hat{f}(\beta) z^{\beta}\right|=\frac{1}{\left|z_{i_{0}}\right|} & \sum_{\beta}\left|\hat{f}(\beta) z^{\beta}\right|\left|z_{i_{0}}\right| \\
& =\frac{1}{\left|z_{i_{0}}\right|} \sum_{\beta}\left|\hat{f}(\beta) z_{1}^{\beta_{1}} \cdots z_{i_{0}}^{\beta_{i_{0}}+1} \cdots z_{k}^{\beta_{k}}\right|=\frac{1}{\left|z_{i_{0}}\right|} \sum_{\alpha}\left|\hat{\tilde{f}}(\alpha) z^{\alpha}\right|<\infty .
\end{aligned}
$$

Hence $z \in \operatorname{mon} H_{p}^{m-1}\left(\mathbb{T}^{\infty}\right)$.

Finally, we are ready to give the

Proof of Theorem 3.1. The case $p=\infty$ follows from Theorem 2.1 and Lemma 3.2 Assume that $1 \leq p<\infty$, and observe that by (35) it suffices to handle the case $p=2$. If $z \in \ell_{2}$ and $f \in H_{2}^{m}\left(\mathbb{T}^{\infty}\right)$, then we apply the Cauchy-Schwarz inequality and the binomial formula to get

$$
\sum_{\substack{\alpha \in \mathbb{N}_{0}^{(\mathbb{N})} \\|\alpha|=m}}\left|\hat{f}(\alpha) z^{\alpha}\right| \leq\left(\sum_{\substack{\alpha \in \mathbb{N}_{0}^{(\mathbb{N})} \\|\alpha|=m}}|\hat{f}(\alpha)|^{2}\right)^{\frac{1}{2}}\left(\sum_{\substack{\alpha \in \mathbb{N}_{0}^{(\mathbb{N})} \\|\alpha|=m}}|z|^{2 \alpha}\right)^{\frac{1}{2}} \leq\|z\|_{2}^{m}\|f\|_{2}<\infty ;
$$


this implies $z \in$ mon $H_{2}^{m}\left(\mathbb{T}^{\infty}\right)$. Conversely, it is enough to show that

$$
\operatorname{mon} H_{2}^{1}\left(\mathbb{T}^{\infty}\right) \subset \ell_{2}
$$

since then by Lemma $3.2 \operatorname{mon} H_{2}^{m}\left(\mathbb{T}^{\infty}\right) \subset \operatorname{mon} H_{2}^{1}\left(\mathbb{T}^{\infty}\right) \subset \ell_{2}$. Fix $z \in \operatorname{mon} H_{2}^{1}\left(\mathbb{T}^{\infty}\right)$. By a closed-graph argument there is $c_{z}>0$ such that for every $f \in H_{2}^{1}\left(\mathbb{T}^{\infty}\right)$ the inequality $\sum_{n=1}^{\infty}\left|\hat{f}(n) z_{n}\right| \leq c_{z}\|f\|_{2}$ holds. Take $y \in \ell_{2}$, and define for each $N$ the function $f_{N}: \mathbb{T}^{\infty} \rightarrow \mathbb{C}, f_{N}(w)=\sum_{n=1}^{N} w_{n} y_{n}$. Clearly, $f_{N} \in H_{2}^{1}\left(\mathbb{T}^{\infty}\right)$ and $\hat{f}_{N}(n)=y_{n}$ for $n=1, \ldots, N$. As a consequence we have

$$
\sum_{n=1}^{N}\left|y_{n} z_{n}\right| \leq c_{z}\left(\sum_{n=1}^{N}\left|y_{n}\right|^{2}\right)^{\frac{1}{2}} \leq c_{z}\|y\|_{2},
$$

and since this holds for every $N$ we by duality see that $z \in \ell_{2}$. Finally, the inequaliy (38) follows immediately from Theorem 2.1 and Lemma 3.2 for the case $p=\infty$. Moreover, (40) gives (38) for $2 \leq p<\infty$ with $C=1$, and 40) combined with Lemma $2.3(s=2$ and $r=1)$ give the inequality with $C \leq \sqrt{2}$ whenever $1 \leq p<2$.

\subsection{The general case}

We address now our main goal of describing mon $H_{p}\left(\mathbb{T}^{\infty}\right)$. There are three significant cases: $p=1, p=2$, and $p=\infty$. The description of mon $H_{p}\left(\mathbb{T}^{\infty}\right)$ for $1 \leq p<\infty$ will follow from the cases $p=1$ and $p=2$, showing that these two coincide.

\section{Theorem 3.4.}

(a) $\mathbf{B} \subset \operatorname{mon} H_{\infty}\left(\mathbb{T}^{\infty}\right) \subset \overline{\mathbf{B}}$

(b) $\operatorname{mon} H_{p}\left(\mathbb{T}^{\infty}\right)=\ell_{2} \cap B_{\ell_{\infty}}$ for $1 \leq p<\infty$.

Again we prepare the proof (which will be given after Lemma 3.7) by some independently interesting observations. Part (a) is an immediate consequence of Theorem 2.2 and the fact that

$$
\operatorname{mon} H_{\infty}\left(\mathbb{T}^{\infty}\right)=\operatorname{mon} H_{\infty}\left(B_{c_{0}}\right)=\operatorname{mon} H_{\infty}\left(B_{\ell_{\infty}}\right),
$$

which we already mentioned without proof in 36): For the second equality see (16) whereas the proof of mon $H_{\infty}\left(\mathbb{T}^{\infty}\right)=\operatorname{mon} H_{\infty}\left(B_{c_{0}}\right)$ is a consequence of the following theorem due to Cole and Gamelin [8, 11.2 Theorem] (see also [18, Lemma 2.3]). For the sake of completeness we include an elementary direct proof; the statement about the inverse mapping seems to be new. 
Proposition 3.5. There exists a unique surjective isometry

$$
\phi: H_{\infty}\left(\mathbb{T}^{\infty}\right) \rightarrow H_{\infty}\left(B_{c_{0}}\right)
$$

such that for every $f \in H_{\infty}\left(\mathbb{T}^{\infty}\right)$ and every $\alpha \in \mathbb{N}_{0}^{(\mathbb{N})}$ we have

$$
c_{\alpha}(\phi(f))=\hat{f}(\alpha) .
$$

Moreover, when restricted to $H_{\infty}^{m}\left(\mathbb{T}^{\infty}\right)$, the mapping $\psi$ defined in Proposition 3.2 and $\phi$ are inverse to each other.

Proof. First of all, let us note that in the finite dimensional setting the result is true: It is a well known fact (see e.g. [27, 3.4.4 exercise (c)]) that for each $n$ there exists an isometric bijection $\phi_{n}: H_{\infty}\left(\mathbb{T}^{n}\right) \rightarrow H_{\infty}\left(\mathbb{D}^{n}\right)$ such that $c_{\alpha}(\phi(f))=\tilde{f}(\alpha)$ for every $f \in H_{\infty}\left(\mathbb{T}^{n}\right)$ and every $\alpha \in \mathbb{N}_{0}^{n}$. Take now $f \in H_{\infty}\left(\mathbb{T}^{\infty}\right)$ and fix $n \in \mathbb{N}$; since we can consider $\mathbb{T}^{\infty}=\mathbb{T}^{n} \times \mathbb{T}^{\infty}$, we write $w=\left(w_{1}, \ldots, w_{n}, \tilde{w}_{n}\right) \in \mathbb{T}^{\infty}$. Then we define $f_{n}: \mathbb{T}^{n} \rightarrow \mathbb{C}$ by

$$
f_{n}\left(w_{1}, \ldots w_{n}\right)=\int_{\mathbb{T} \infty} f\left(w_{1}, \ldots, w_{n}, \tilde{w}_{n}\right) d m\left(\tilde{w}_{n}\right) .
$$

By the Fubini theorem $f_{n}$ is well defined a.e. and

$$
\int_{\mathbb{T}^{\infty}} f(w) d m(w)=\int_{\mathbb{T}^{n}}\left(\int_{\mathbb{T}^{\infty}} f\left(w_{1}, \ldots, w_{n}, \tilde{w}_{n}\right) d m\left(\tilde{w}_{n}\right)\right) d m_{n}\left(w_{1}, \ldots, w_{n}\right),
$$

hence $f_{n} \in L_{\infty}\left(\mathbb{T}^{n}\right)$. Moreover, for each $\alpha \in \mathbb{Z}^{n}$ we have, again by Fubini's theorem,

$$
\hat{f}_{n}(\alpha)=\int_{\mathbb{T}^{n} \times \mathbb{T}} f(w) w^{-\alpha} d m(w)=\hat{f}(\alpha) .
$$

and hence $\hat{f}_{n}(\alpha)=\hat{f}(\alpha)=0$ for every $\alpha \in \mathbb{Z}^{n} \backslash \mathbb{N}_{0}^{n}$. Since obviously $\left\|f_{n}\right\|_{\infty} \leq\|f\|_{\infty}$ (the measure $d m$ is a probability) we obtain that $f_{n} \in H_{\infty}\left(\mathbb{T}^{n}\right)$, and can now consider $g_{n}=\phi_{n}\left(f_{n}\right) \in H_{\infty}\left(\mathbb{D}^{n}\right)$. We have $\left\|g_{n}\right\|_{\infty}=\left\|f_{n}\right\|_{\infty} \leq\|f\|_{\infty}$ and

$$
g_{n}(z)=\sum_{\alpha \in \mathbb{N}_{0}^{n}} \hat{f}_{n}(\alpha) z^{\alpha}=\sum_{\alpha \in \mathbb{N}_{0}^{n}} \hat{f}(\alpha) z^{\alpha}
$$

for every $z \in \mathbb{D}^{n}$. Since this holds for every $n$ we define $g: \mathbb{D}^{(\mathbb{N})} \rightarrow \mathbb{C}$ by $g(z)=$ $\sum_{\alpha \in \mathbb{N}_{0}^{n}} \hat{f}(\alpha) z^{\alpha}$. We have $\|g\|_{\infty}=\sup _{n}\left\|g_{n}\right\|_{\infty} \leq\|f\|_{\infty}$. By [12, Lemma 2.2] there exists a unique extension $\tilde{g} \in H_{\infty}\left(B_{c_{0}}\right)$ with $c_{\alpha}(\tilde{g})=\hat{f}(\alpha)$ and $\|\tilde{g}\|_{\infty}=\|g\|_{\infty} \leq$ $\|f\|_{\infty}$. Setting $\phi(f)=\tilde{g}$ we see that $\phi: H_{\infty}\left(\mathbb{T}^{\infty}\right) \rightarrow H_{\infty}\left(B_{c_{0}}\right)$ is a well defined linear mapping such that for every $f \in H_{\infty}\left(\mathbb{T}^{\infty}\right)$ and every $\alpha \in \mathbb{N}_{0}^{(\mathbb{N})}$ we have $\|\phi(f)\|_{\infty} \leq\|f\|_{\infty}$ and $c_{\alpha}(\phi(f))=\tilde{f}(\alpha)$. On the other hand, if $f \in L_{\infty}\left(\mathbb{T}^{\infty}\right)$ is such that $\hat{f}(\alpha)=0$ for all $\alpha$, then $f=0$. Hence $\phi$ is injective. 
Let us see that it is also surjective and moreover an isometry. Fix $g \in H_{\infty}\left(B_{c_{0}}\right)$ and consider $g_{n}$ its restriction to the first $n$ variables. Clearly $g_{n} \in H_{\infty}\left(\mathbb{D}^{n}\right)$ and $\left\|g_{n}\right\|_{\infty} \leq\|g\|_{\infty}$. Using again [27, 3.4.4 exercise (c)] we can choose $f_{n} \in H_{\infty}\left(\mathbb{T}^{n}\right)$ such that $\left\|f_{n}\right\|_{\infty}=\left\|g_{n}\right\|_{\infty}$ and $c_{\alpha}\left(g_{n}\right)=\hat{f}_{n}(\alpha)$ for all $\alpha \in \mathbb{N}_{0}^{n}$. Since $c_{\alpha}\left(g_{n}\right)=$ $c_{\alpha}(g)$, we have $\hat{f}_{n}(\alpha)=c_{\alpha}(g)$ for all $\alpha \in \mathbb{N}_{0}^{n}$. We define now $\tilde{f}_{n} \in H_{\infty}\left(\mathbb{T}^{\infty}\right)$ by $\tilde{f}_{n}(w)=f_{n}\left(w_{1}, \ldots, w_{n}\right)$ for $w \in \mathbb{T}^{\infty}$. Then the sequence $\left(\tilde{f}_{n}\right)_{n=1}^{\infty}$ is contained in the closed ball in $L_{\infty}\left(\mathbb{T}^{\infty}\right)$ centered at 0 and with radius $\|g\|_{\infty}$. Since this ball is $w^{*}$-compact and metrizable, there is a subsequence $\left(\tilde{f}_{n_{k}}\right)_{k}$ that $w^{*}$-converges to some $f \in L_{\infty}\left(\mathbb{T}^{\infty}\right)$ with $\|f\|_{\infty} \leq\|g\|_{\infty}$. Moreover,

$$
\hat{f}(\alpha)=\left\langle f, w^{\alpha}\right\rangle=\lim _{k \rightarrow \infty}\left\langle\tilde{f}_{n_{k}}, w^{\alpha}\right\rangle=\lim _{k \rightarrow \infty} \hat{\tilde{f}}_{n_{k}}(\alpha)
$$

for every $\alpha \in \mathbb{Z}_{0}^{(\mathbb{N})}$, and this implies $f \in H_{\infty}\left(\mathbb{T}^{\infty}\right)$. Let us see that $\phi(f)=g$, which shows that $\phi$ is onto; indeed, if $\alpha=\left(\alpha_{1}, \ldots, \alpha_{n_{0}}, 0, \ldots\right)$ then for $n_{k} \geq n_{0}$ we have

$$
\left\langle\tilde{f}_{n_{k}}, w^{\alpha}\right\rangle=\int_{\mathbb{T} \infty} \tilde{f}_{n_{k}}(w) w^{-\alpha} d m(w)=\int_{\mathbb{T}^{n_{k}}} f_{n_{k}}(w) w^{-\alpha} d m_{n_{k}}(w)=\hat{f}_{n_{k}}(\alpha)=c_{\alpha}(g) .
$$

Hence $\hat{f}(\alpha)=c_{\alpha}(g)$ for all $\alpha \in \mathbb{N}_{0}^{(\mathbb{N})}$. Furthermore, since $\|f\|_{\infty} \leq\|g\|_{\infty}=\|\phi(f)\|_{\infty}$ we also get that $\phi$ is an isometry. Let us fix $P \in \mathscr{P}\left({ }^{m} c_{0}\right)$ and show that $\phi^{-1}(P)(w)=$ $\tilde{P}(w)$ for every $w \in \mathbb{T}^{\infty}$. We choose $\left(J_{k}\right)_{k}$ a sequence of finite families of multiindexes included in $\left\{\alpha: \alpha \in \mathbb{N}_{0}^{(\mathbb{N})}:|\alpha|=m\right\}$ and such that the sequence $P_{k}=$ $\sum_{\alpha \in J_{k}} c_{\alpha, k} x^{\alpha}$ converges uniformly to $P$ on the unit ball of $c_{0}$. Since each $J_{k}$ is finite, we have

$$
\phi^{-1}\left(P_{k}\right)(w)=\sum_{\alpha \in J_{k}} c_{\alpha, k} w^{\alpha}=\tilde{P}_{k}(w),
$$

for every $w \in \mathbb{T}^{\infty}$. The linearity of the $A B$ operator and (39) give that $\left\|\tilde{P}-\tilde{P}_{k}\right\|=$ $\left\|P-P_{k}\right\|=\left\|\phi^{-1}(P)-\phi^{-1}\left(P_{k}\right)\right\|$ converges to 0 and complete the proof.

Observe that this argument actually works to prove that $\phi^{-1}(g)(w)=\tilde{g}(w)$ for every $w \in \mathbb{T}^{\infty}$ and every function $g$ in the completion of the space of all polynomials on $c_{0}$.

We handle now the case $p=2$ of part (b) of Theorem 3.4 where slightly more can be said (for the proof of Theorem 3.4 this will not be needed). Here, since $H_{2}\left(\mathbb{T}^{\infty}\right)$ is a Hilbert space with the orthonormal basis $\left\{w^{\alpha}\right\}_{\alpha}$, we have $\|f\|_{2}=$ $\left(\sum_{\alpha}|\hat{f}(\alpha)|^{2}\right)^{1 / 2}$ which simplifies the problem a lot.

Proposition 3.6. We have

$$
\operatorname{mon} H_{2}\left(\mathbb{T}^{\infty}\right)=\ell_{2} \cap B_{\ell_{\infty}} \text {, }
$$


and for each $z \in \ell_{2} \cap B_{\ell_{\infty}}$ and $f \in H_{2}\left(\mathbb{T}^{\infty}\right)$,

$$
\sum_{\alpha \in \mathbb{N}_{0}^{(N)}}\left|\hat{f}(\alpha) z^{\alpha}\right| \leq\left(\prod_{n=1}^{\infty} \frac{1}{1-\left|z_{n}\right|^{2}}\right)^{\frac{1}{2}}\|f\|_{2} .
$$

Moreover, the constant $\left(\prod_{n} \frac{1}{1-\left|z_{n}\right|^{2}}\right)^{1 / 2}$ is optimal.

Proof. The fact that $\ell_{2} \cap B_{\ell_{\infty}} \subset$ mon $H_{2}\left(\mathbb{T}^{\infty}\right)$ follows by using the Cauchy-Schwarz inequality in a similar way as in (40):

$$
\sum_{\alpha \in \mathbb{N}_{0}^{(\mathbb{N})}}\left|\hat{f}(\alpha) z^{\alpha}\right| \leq\left(\sum_{\alpha \in \mathbb{N}_{0}^{(N)}}|\hat{f}(\alpha)|^{2}\right)^{\frac{1}{2}}\left(\sum_{\alpha \in \mathbb{N}_{0}^{(N)}}|z|^{2 \alpha}\right)^{\frac{1}{2}}=\|f\|_{2}\left(\prod_{n=1}^{\infty} \frac{1}{1-\left|z_{n}\right|^{2}}\right)^{\frac{1}{2}}<\infty .
$$

On the other hand, since $H_{2}^{1}\left(\mathbb{T}^{\infty}\right) \subset H_{2}\left(\mathbb{T}^{\infty}\right)$ we have that mon $H_{2}\left(\mathbb{T}^{\infty}\right)$ is a subset of mon $H_{2}^{1}\left(\mathbb{T}^{\infty}\right)$, and hence Theorem 3.1 gives the conclusion. Let us see that for each fixed $z \in$ mon $H_{2}\left(\mathbb{T}^{\infty}\right)=\ell_{2} \cap B_{\ell_{\infty}}$ the constant in (41) is optimal: By a closed graph argument there is $c_{z}>0$ such that for all $f \in H_{2}\left(\mathbb{T}^{\infty}\right)$

$$
\sum_{\alpha \in \mathbb{N}_{0}^{(N)}}\left|\hat{f}(\alpha) z^{\alpha}\right| \leq c_{z}\|f\|_{2},
$$

and moreover

$$
\sum_{\alpha \in \mathbb{N}_{0}^{(\mathbb{N})}}|z|^{2 \alpha}<\infty
$$

Then the function $f_{z}(w)=\sum_{\alpha \in \mathbb{N}_{0}^{(\mathbb{N})}} z^{\alpha} w^{\alpha} \in H_{2}\left(\mathbb{T}^{\infty}\right)$ is well defined and $\hat{f}_{z}(\alpha)=$ $z^{\alpha}$ for every $\alpha \in \mathbb{N}_{0}^{(\mathbb{N})}$ (and 0 otherwise). Hence

$$
\sum_{\alpha \in \mathbb{N}_{0}^{(\mathbb{N})}}\left|z^{\alpha}\right|^{2}=\sum_{\alpha \in \mathbb{N}_{0}^{(\mathbb{N})}}\left|\hat{f}_{z}(\alpha) z^{\alpha}\right| \leq c\left\|f_{z}\right\|_{2}=c\left(\sum_{\alpha \in \mathbb{N}_{0}^{(\mathbb{N})}}\left|z^{\alpha}\right|^{2}\right)^{\frac{1}{2}},
$$

which completes the proof.

In order to extend this result to the general case $1 \leq p<\infty$ we need another important lemma - an $H_{p}$-version of [6, Satz VI] (see also [13, Lemma 2]).

Lemma 3.7. Let $z \in \operatorname{mon} H_{p}\left(\mathbb{T}^{\infty}\right)$ and $x=\left(x_{n}\right)_{n} \in B_{\ell_{\infty}}$ such that $\left|x_{n}\right| \leq\left|z_{n}\right|$ for all but finitely many $n$ 's. Then $x \in$ mon $H_{p}\left(\mathbb{T}^{\infty}\right)$.

Proof. We follow [13, Lemma 2] and choose $r \in \mathbb{N}$ such that $\left|x_{n}\right| \leq\left|z_{n}\right|$ for all $n>r$. We also take $a>1$ such that $\left|z_{n}\right|<\frac{1}{a}$ for $n=1, \ldots, r$. Let $f \in H_{p}\left(\mathbb{T}^{\infty}\right)$ with $\|f\|_{p} \leq 1$. We fix $n_{1}, \ldots, n_{r} \in \mathbb{N}$ and define for each $u \in \mathbb{T}^{\infty}$,

$$
f_{n_{1}, \ldots, n_{r}}(u)=\int_{\mathbb{T} r} f\left(w_{1}, \ldots, w_{r}, u_{1}, \ldots\right) w_{1}^{-n_{1}} \cdots w_{r}^{-n_{r}} d m_{r}\left(w_{1}, \ldots, w_{r}\right) .
$$


Let us see that $f_{n_{1}, \ldots, n_{r}} \in H_{p}\left(\mathbb{T}^{\infty}\right)$; indeed, using Hölder's inequality we have

$$
\begin{aligned}
& \left(\int_{\mathbb{T}^{\infty}}\left|f_{n_{1}, \ldots, n_{r}}(u)\right|^{p} d m(u)\right)^{\frac{1}{p}} \\
& =\left(\int_{\mathbb{T}^{\infty}}\left|\int_{\mathbb{T}^{r}} f\left(w_{1}, \ldots, w_{r}, u_{1}, \ldots\right) w_{1}^{-n_{1}} \cdots w_{r}^{-n_{r}} d m_{r}\left(w_{1}, \ldots, w_{r}\right)\right|^{p} d m(u)\right)^{\frac{1}{p}} \\
& \leq\left(\int_{\mathbb{T}^{\infty}}\left(\int_{\mathbb{T}^{r}}\left|f\left(w_{1}, \ldots, w_{r}, u_{1}, \ldots\right)\right|^{p} d m_{r}\left(w_{1}, \ldots, w_{r}\right)\right) d m(u)\right)^{\frac{1}{p}}=\|f\|_{p} .
\end{aligned}
$$

Hence $f_{n_{1}, \ldots, n_{r}} \in L_{p}\left(\mathbb{T}^{\infty}\right)$ and $\left\|f_{n_{1}, \ldots, n_{r}}\right\|_{p} \leq\|f\|_{p} \leq 1$. Now we have, for every multi index $\alpha=\left(\alpha_{1}, \ldots, \alpha_{k}, 0, \ldots\right)$

$$
\begin{aligned}
& \hat{f}_{n_{1}, \ldots, n_{r}}(\alpha) \\
& =\int_{\mathbb{T} \infty} f_{n_{1}, \ldots, n_{r}}(u) u^{-\alpha} d m(u) \\
& =\left(\int_{\mathbb{T} \infty} \int_{\mathbb{T} r} \frac{f\left(w_{1}, \ldots, w_{r}, u_{1}, \ldots, u_{k}\right)}{w_{1}^{n_{1}} \cdots w_{r}^{n_{r}} u_{1}^{\alpha_{1}} \cdots u_{k}^{\alpha_{k}}} d m_{r}\left(w_{1}, \ldots, w_{r}\right) d m_{k}\left(u_{1}, \ldots, u_{k}, 0, \ldots\right)\right) \\
& =\hat{f}\left(n_{1}, \ldots, n_{r}, \alpha_{1}, \ldots, \alpha_{k}, 0, \ldots\right) .
\end{aligned}
$$

Therefore

$$
\hat{f}_{n_{1}, \ldots, n_{r}}(\alpha)= \begin{cases}\hat{f}\left(n_{1}, \ldots, n_{r}, \alpha_{1}, \ldots, \alpha_{k}, 0, \ldots\right) & \text { if } \alpha=\left(0, ., ., 0, \alpha_{1}, \ldots \alpha_{k}, 0, \ldots\right) \\ 0 & \text { otherwise }\end{cases}
$$

and this implies $f_{n_{1}, \ldots, n_{r}} \in H_{p}\left(\mathbb{T}^{\infty}\right)$. Now, using (43) (below) and doing exactly the same calculations as in [13, Lemma 2] we conclude $\sum_{\alpha}\left|\hat{f}(\alpha) x^{\alpha}\right|<\infty$ and $x$ belongs to mon $H_{p}\left(\mathbb{T}^{\infty}\right)$.

Finally, we are ready for the

Proof of Theorem 3.4- $[$ b). Lower inclusion: Let us remark first that

$$
\text { mon } H_{1}\left(\mathbb{T}^{\infty}\right) \subset \operatorname{mon} H_{p}\left(\mathbb{T}^{\infty}\right)
$$

since $H_{p}\left(\mathbb{T}^{\infty}\right) \subset H_{1}\left(\mathbb{T}^{\infty}\right)$. Then to get the lower bound it is enough to show that $\ell_{2} \cap B_{\ell_{\infty}} \subset$ mon $H_{1}\left(\mathbb{T}^{\infty}\right)$. As a first step we show that there exists $0<r<1$ such that $r B_{\ell_{2}} \cap B_{\ell_{\infty}} \subset$ mon $H_{1}\left(\mathbb{T}^{\infty}\right)$. Let $r<1 / \sqrt{2}$ and $z \in r B_{\ell_{2}} \cap B_{\ell_{\infty}}$. Then $z=r y$ for some $y \in B_{\ell_{2}}$. By [8, 9.2 Theorem] there exists a contractive projection $P_{m}: H_{1}\left(\mathbb{T}^{\infty}\right) \rightarrow H_{1}^{m}\left(\mathbb{T}^{\infty}\right)$ such that $\widehat{P_{m} g}(\alpha)=\hat{g}(\alpha)$ whenever $|\alpha|=m$ and 0 otherwise. Then for every $f \in H_{1}\left(\mathbb{T}^{\infty}\right)$ with $f_{m}=P_{m}(f)$ we obtain

$$
\begin{aligned}
\sum_{\alpha}\left|\hat{f}(\alpha) z^{\alpha}\right|=\sum_{m=0}^{\infty} \sum_{|\alpha|=m}\left|\hat{f}(\alpha)(r y)^{\alpha}\right| & =\sum_{m=0|\alpha|=m}^{\infty}\left|\hat{f}_{m}(\alpha)(r y)^{\alpha}\right| \\
\leq & \sum_{m=0}^{\infty} r^{m}(\sqrt{2})^{m}\left\|f_{m}\right\|_{1} \leq \sum_{m=0}^{\infty}\left(r \sqrt{2}^{m}\|f\|_{1}<\infty,\right.
\end{aligned}
$$


where in the first inequality we used that $y \in \ell_{2}$ and (38), and in the second one the fact that $\left\|P_{m}\right\| \leq 1$. Take now some $z \in \ell_{2} \cap B_{\ell_{\infty}}$. Then $\left(\sum_{n=n_{0}}^{\infty}\left|z_{n}\right|^{2}\right)^{1 / 2}<r$ for some $n_{0}$, and we define

$$
x=\left(0, \ldots, 0, z_{n_{0}}, z_{n_{0}+1}, \ldots\right) \in r B_{\ell_{2}} \cap B_{\ell_{\infty}} .
$$

As explained $x \in$ mon $H_{1}\left(\mathbb{T}^{\infty}\right)$, and hence Lemma 3.7 implies as desired $z \in$ mon $H_{1}\left(\mathbb{T}^{\infty}\right)$. Upper inclusion: Again by 2.3 we have $H_{p}^{1}\left(\mathbb{T}^{\infty}\right)=H_{2}^{1}\left(\mathbb{T}^{\infty}\right)$ with equivalent norms. This, together with Theorem 3.1, gives

$$
\operatorname{mon} H_{p}\left(\mathbb{T}^{\infty}\right) \subset \operatorname{mon} H_{p}^{1}\left(\mathbb{T}^{\infty}\right)=\operatorname{mon} H_{2}^{1}\left(\mathbb{T}^{\infty}\right) \subset \ell_{2} \cap B_{\ell_{\infty}} \text {. }
$$

Remark 3.8. Denote by $\mathscr{P}_{\text {fin }}$ the space of all trigonometric polynomials on $\mathbb{C}^{\mathbb{N}}$ (all finite sums $\sum_{\alpha \in J} c_{\alpha} z^{\alpha}$ ). For each $z \in \ell_{\infty}$ the evaluation mapping

$$
\delta_{z}: \mathscr{P}_{\mathrm{fin}} \rightarrow \mathbb{C}, \delta_{z}(f)=f(z)
$$

is clearly well defined. One of the main problems considered in [8] is to determine for which $z$ 's the evaluation mapping $\delta_{z}$ extends continuously to the whole space $H_{p}\left(\mathbb{T}^{\infty}\right), 1 \leq p<\infty$. This can be reformulated as to describe the following set

$$
\left\{z \in \ell_{\infty}\left|\exists c_{z}>0 \forall f \in \mathscr{P}_{\text {fin }}:\right| f(z) \mid \leq c_{z}\|f\|_{p}\right\} .
$$

Since for each $f \in \mathscr{P}_{\text {fin }}$ and every $\alpha$ we have $\hat{f}(\alpha)=c_{\alpha}$, the previous set can be written as

$$
\left\{z \in \ell_{\infty}\left|\exists c_{z}>0 \forall f \in \mathscr{P}_{\text {fin }}:\right| \sum_{\alpha} \hat{f}(\alpha) z^{\alpha} \mid \leq c_{z}\|f\|_{p}\right\} .
$$

In [8, 8.1 Theorem] it is shown that for $1 \leq p<\infty$ the set in (42) is exactly $\ell_{2} \cap$ $B_{\ell_{\infty}}$. By a simple closed-graph argument, for each $1 \leq p<\infty$ a sequence $z$ belongs to the set mon $H_{p}\left(\mathbb{T}^{\infty}\right)$ if and only if there exists $c_{z}>0$ such that for every $f \in H_{p}\left(\mathbb{T}^{\infty}\right)$

$$
\sum_{\alpha \in \mathbb{N}_{0}^{(\mathbb{N})}}\left|\hat{f}(\alpha) z^{\alpha}\right| \leq c_{z}\left(\int_{\mathbb{T}^{\infty}}|f(w)|^{p} d m(w)\right)^{\frac{1}{p}} .
$$

This implies

$$
\operatorname{mon} H_{p}\left(\mathbb{T}^{\infty}\right)=\left\{z \in \ell_{\infty}\left|\exists c_{z}>0 \forall f \in \mathscr{P}_{\mathrm{fin}}: \sum_{\alpha}\right| \hat{f}(\alpha) z^{\alpha} \mid \leq c_{z}\|f\|_{p}\right\} .
$$

In view of (44) we have that mon $H_{p}\left(\mathbb{T}^{\infty}\right)$ is contained in the set in (42). Then the upper inclusion in Theorem 3.4 (b) follows from [8, 8.1 Theorem]. The proof we presented here is independent from that in [8]. But the lower inclusion in Theorem 3.4 (b) is stronger than the result in [8]. 


\subsection{Representation of Hardy spaces}

We have seen in Proposition 3.5 how, like in the finitely dimensional case, the Hardy space $H_{\infty}\left(\mathbb{T}^{\infty}\right)$ can be represented as a space of holomorphic functions on $c_{0}$. In [8, 10.1 Theorem] it is proved that every function $f \in H_{p}\left(\mathbb{T}^{\infty}\right)$ uniquely determines a holomorphic function $g$ of bounded type on the Reinhardt domain $B_{\ell_{\infty}} \cap \ell_{2}$ in $\ell_{2}$ such that the Fourier coefficients $\hat{f}(\alpha)$ of $f$ coincide with the monomial coefficients $c_{\alpha}$ (g) of $g$ (recall that $g$ is of bounded type on $R$ if it is bounded on bounded sets contained in $R$ with positive distance to $\ell_{2} \backslash R$ ). In the following we use our new knowledge on mon $H_{p}\left(\mathbb{T}^{\infty}\right)$ in order to characterize all those $g$ 's coming from functions $f \in H_{p}\left(\mathbb{T}^{\infty}\right)$. For the Reinhardt domain $R=B_{\ell_{\infty}} \cap \ell_{2}$ in $\ell_{2}$ define the Banach space

$$
H_{p}(R)=H_{p}\left(B_{\ell_{\infty}} \cap \ell_{2}\right)
$$

of all holomorphic functions $g: R \rightarrow \mathbb{C}$ for which

$$
\|g\|_{H_{p}(R)}=\sup _{n \in \mathbb{N}} \sup _{0<r<1}\left(\int_{\mathbb{T}^{n}}\left|g\left(r w_{1}, \ldots, r w_{n}, 0,0, \ldots\right)\right|^{p} d m_{n}\left(w_{1}, \ldots, w_{n}\right)\right)^{\frac{1}{p}}<\infty .
$$

Theorem 3.9. For each $1 \leq p<\infty$ the mapping

$$
\phi: H_{p}\left(\mathbb{T}^{\infty}\right) \rightarrow H_{p}\left(B_{\ell_{\infty}} \cap \ell_{2}\right)
$$

defined by

$$
\phi(f)(z)=\sum_{\alpha \in \mathbb{N}_{0}^{(\mathbb{N})}} \hat{f}(\alpha) z^{\alpha}, z \in B_{\ell_{\infty}} \cap \ell_{2}
$$

is an onto isometry.

Proof. Let us begin by noting that for each fixed $n$ the mapping

$$
\phi_{n}: H_{p}\left(\mathbb{T}^{n}\right) \rightarrow H_{p}\left(\mathbb{D}^{n}\right), \phi_{n}(f)(z)=\sum_{\alpha \in \mathbb{N}_{0}^{n}} \hat{f}(\alpha) z^{\alpha}
$$

is an isometric isomorphism, where $H_{p}\left(\mathbb{D}^{n}\right)$ denotes the Banach space of all holomorphic functions $g: \mathbb{D}^{n} \rightarrow \mathbb{C}$ such that

$$
\|g\|_{H_{p}\left(\mathbb{D}^{n}\right)}=\sup _{0<r<1}\left(\int_{\mathbb{T}^{n}}\left|g\left(r w_{1}, \ldots, r w_{n}\right)\right|^{p} d m_{n}\left(w_{1}, \ldots, w_{n}\right)\right)^{\frac{1}{p}}<\infty .
$$

We show in first place that $\phi$ is well defined and a contraction. Fix $f \in H_{p}\left(\mathbb{T}^{\infty}\right)$; we know from Theorem 3.4 that $\sum_{\left.\alpha \in \in \mathbb{N}_{0}^{(\mathbb{N}}\right)}\left|\hat{f}(\alpha) z^{\alpha}\right|<\infty$ for every $z \in B_{\ell_{\infty}} \cap \ell_{2}$, 
hence the series defines a Gâteaux-differentiable function on $B_{\ell_{\infty}} \cap \ell_{2}$. We denote the $m$-th Taylor polynomial of $\phi(f)$ at 0 by $P_{m}$. Since for all $z \in \ell_{2}$

$$
P_{m}(z)=\sum_{\substack{\alpha \in \mathbb{N}_{0}^{n} \\|\alpha|=m}} \hat{f}(\alpha) z^{\alpha},
$$

we deduce from (38) that $P \in \mathscr{P}\left({ }^{m} \ell_{2}\right)$, and hence $\phi(f)$ defines a holomorphic function on $B_{\ell_{\infty}} \cap \ell_{2}$ (see e.g. [15, Example 3.8]). Let us see now that it actually belongs to $H_{p}\left(B_{\ell_{\infty}} \cap \ell_{2}\right)$. Following the notation in Proposition 3.5 we define for each $n$

$$
f_{n}\left(w_{1}, \ldots, w_{n}\right)=\int_{\mathbb{T} \infty} f\left(w_{1}, \ldots, w_{n}, \tilde{w}_{n}\right) d m\left(\tilde{w}_{n}\right),
$$

where $\left(w_{1}, \ldots, w_{n}\right) \in \mathbb{T}^{n}$. By Fubini's theorem and since $L_{p}\left(\mathbb{T}^{\infty}\right) \subset L_{1}\left(\mathbb{T}^{\infty}\right)$, this function is well defined. On the other hand, using Hölder's inequality and again Fubini's theorem we get

$$
\begin{aligned}
\int_{\mathbb{T}^{n}} & \left|f_{n}\left(w_{1}, \ldots, w_{n}\right)\right|^{p} d m_{n}\left(w_{1}, \ldots, w_{n}\right) \\
\quad & \int_{\mathbb{T}^{n}}\left|\int_{\mathbb{T}^{\infty}} f\left(w_{1}, \ldots, w_{n}, \tilde{w}_{n}\right) d m\left(\tilde{w}_{n}\right)\right|^{p} d m_{n}\left(w_{1}, \ldots, w_{n}\right) \\
\quad & \int_{\mathbb{T}^{n}}\left(\int_{\mathbb{T}^{\infty}}\left|f\left(w_{1}, \ldots, w_{n}, \tilde{w}_{n}\right)\right| d m\left(\tilde{w}_{n}\right)\right)^{p} d m_{n}\left(w_{1}, \ldots, w_{n}\right) \\
& \leq \int_{\mathbb{T}^{n}}\left(\int_{\mathbb{T}^{\infty}}\left|f\left(w_{1}, \ldots, w_{n}, \tilde{w}_{n}\right)\right|^{p} d m\left(\tilde{w}_{n}\right)\right) d m_{n}\left(w_{1}, \ldots, w_{n}\right),
\end{aligned}
$$

and this implies $f \in L_{p}\left(\mathbb{T}^{n}\right)$ and $\left\|f_{n}\right\|_{p} \leq\|f\|_{p}$ for all $n$. Moreover, for $\alpha \in \mathbb{Z}^{n}$ we have (again using Fubini) $\hat{f}_{n}(\alpha)=\hat{f}(\alpha)$ and $f_{n} \in H_{p}\left(\mathbb{T}^{n}\right)$. Then $\left\|\phi\left(f_{n}\right)\right\|_{H_{p}\left(\mathbb{D}^{n}\right)}=$ $\left\|f_{n}\right\|_{p} \leq\|f\|_{p}$ for all $n$, and we arrive at

$$
\sup _{n \in \mathbb{N}} \sup _{0<r<1} \int_{\mathbb{J}^{n}}\left|\sum_{\alpha \in \mathbb{N}_{0}^{n}} \hat{f}(\alpha)(r w)^{\alpha}\right| d m_{n}\left(w_{1}, \ldots, w_{n}\right) \leq\|f\|_{p}<\infty .
$$

Clearly, $\phi(f)\left(z_{1}, \ldots, z_{n}, 0 \ldots\right)=\sum_{\alpha} \hat{f}(\alpha) z^{\alpha}$ for every $\left(z_{1}, \ldots, z_{n}\right) \in \mathbb{D}^{n}$, and by (45) this implies

$$
\phi(f) \in H_{p}\left(B_{\ell_{\infty}} \cap \ell_{2}\right) \text { and }\|\phi(f)\|_{H_{p}\left(B_{\ell_{\infty}} \cap \ell_{2}\right)} \leq\|f\|_{p} .
$$

Finally, we show that $\Phi$ is also an isometry onto: Fix some $g \in H_{p}\left(B_{\ell_{\infty}} \cap \ell_{2}\right)$, and denote by $g_{n}$ its restriction to the first $n$ variables. Then, by definition $g_{n} \in$ $H_{p}\left(\mathbb{D}^{n}\right)$ and $\left\|g_{n}\right\|_{H_{p}\left(\mathbb{D}^{n}\right)} \leq\|g\|_{H_{p}\left(B_{\ell_{\infty}} \cap \ell_{2}\right)}$. Let us take $f_{n}=\phi_{n}^{-1}\left(g_{n}\right) \in H_{p}\left(\mathbb{T}^{n}\right)$ and define

$$
\tilde{f}_{n}: \mathbb{T}^{\infty} \rightarrow \mathbb{C}, \tilde{f}_{n}(w)=f_{n}\left(w_{1}, \ldots, w_{n}\right) .
$$


Since we can do this for every $n$, we have a sequence $\left(\tilde{f}_{n}\right)_{n}$ contained in the closed ball of $L_{p}\left(\mathbb{T}^{\infty}\right)$ centered in 0 and with radius $\|g\|_{H_{p}\left(B_{\ell_{\infty}} \cap \ell_{2}\right)}$, that is a weak- $\left(L_{p}, L_{q}\right)$-compact set if $1<p<\infty$ ( $q$ the conjugate index of $p$ ). Since $L_{q}\left(\mathbb{T}^{\infty}\right)$ is separable, the weak ${ }^{*}$-topology is metrizable, and hence there exists a subsequence $\left(\tilde{f}_{n_{k}}\right)_{k}$ that weak* converges to some $f \in L_{p}\left(\mathbb{T}^{\infty}\right)$. For each $\alpha \in \mathbb{Z}^{(\mathbb{N})}$ we then have

$$
\hat{f}(\alpha)=\left\langle f, w^{\alpha}\right\rangle=\lim _{k}\left\langle\tilde{f}_{n_{k}}, w^{\alpha}\right\rangle=\hat{\tilde{f}}_{n_{k}}(\alpha)=c_{\alpha}(g) .
$$

Hence $f \in H_{p}\left(\mathbb{T}^{\infty}\right), \phi(f)=g$ and, moreover, $\|f\|_{p} \leq\|g\|_{H_{p}\left(B_{\ell_{\infty}} \cap \ell_{2}\right)}$. This completes the proof for $1<p<\infty$, and it remains to check the case $p=1$ : Using Riesz' representation theorem (for the dual of $C\left(\mathbb{T}^{\infty}\right)$ ), we only obtain that there exists a subsequence $\left(\tilde{f}_{n_{k}}\right)_{k}$ that weak* converges to some complex measure $v$ on $\mathbb{T}^{\infty}$. But as in (46), we have $\hat{v}(\alpha)=c_{\alpha}(g)$ for every $\alpha \in \mathbb{Z}^{(\mathbb{N})}$. In particular, $\hat{v}(\alpha)=0$ for every $\alpha \in \mathbb{Z}^{(\mathbb{N})} \backslash \mathbb{N}_{0}^{(\mathbb{N})}$, i.e., $v$ is an analytic measure. But in [19] it is proved that any analytic measure on a topological group is absolutely continuous with respect to the corresponding Haar measure that in our case is $d m$. Hence, $v$ can be represented by an element $f \in H_{1}\left(\mathbb{T}^{\infty}\right)$, and we hence have, exactly as above, $\phi(f)=g$ and $\|f\|_{1} \leq\|g\|_{H_{1}\left(B_{\ell_{\infty}} \cap \ell_{2}\right)}$.

We want to thank Jan-Fredrik Olsen and Eero Saksman who very recently informed us about the theorem of Helson-Lowdenslager from [19] (the M. and F. Riesz theorem for topological groups) which in the preceding proof was essential to handle the case $p=1$. Actually they, together with A. Aleman, in [1, Corollary 1] give a direct proof of that result for the infinite dimensional polytorus. In [1, Corollary 3] they apply their result to obtain a variant of Theorem 3.9.

\section{$4 \ell_{1}$-multipliers of $\mathscr{H}_{p}$-Dirichlet series}

Finally, we come back to one of our original motivations. We use Bohr's transform from (4) to deduce from our main results on sets of monomial convergence (see 2.1, 2.2, 3.1, and 3.4 multiplier theorems for spaces of Dirichlet series.

Historically all results on sets of monomial convergence (at least those of (8), (17), (18), and (20) were motivated through the theory of Dirichlet series. Maximal domains where such Dirichlet series $D=\sum_{n} a_{n} n^{-s}$ converge conditionally, uniformly or absolutely are half planes $[\operatorname{Re}>\sigma]$, where $\sigma=\sigma_{c}, \sigma_{u}$ or $\sigma_{a}$ are called the abscissa of conditional, uniform or absolute convergence, respectively. More precisely, $\sigma_{\alpha}(D)$ is the infimum of all $r \in \mathbb{R}$ such that on $[\operatorname{Re}>r]$ we have convergence of $D$ of the requested type $\alpha=c, u$ or $a$. Each Dirichlet series $D$ defines a holomorphic function $d:\left[\operatorname{Re}>\sigma_{c}\right] \rightarrow \mathbb{C}$. If $\sigma_{b}(D)$ denotes the 
abscissa of boundedness, i.e. the infimum of all $r \in \mathbb{R}$ such that $d$ on the half plane $[\operatorname{Re}>r]$ is bounded, then one of the fundamental theorems of Bohr from [7] is

$$
\sigma_{u}(D)=\sigma_{b}(D) .
$$

Bohr's so called absolute convergence problem from [6] asked for the largest possible width of the strip in $\mathbb{C}$ on which a Dirichlet series may converge uniformly but not absolutely. In other terms, Bohr defined the number

$$
S:=\sup _{D} \sigma_{a}(D)-\sigma_{u}(D),
$$

where the supremum is taken over all possible Dirichlet series $D$, and asked for its precise value.

Using the prime number theorem Bohr in [6] proved that $S=\frac{1}{M}$, and concluded from (17) that $S \leq 1 / 2$ (for the definition of $M$ see again (19)). Shortly after that Toeplitz with his result from (8) got $1 / 4 \leq S \leq 1 / 2$. Although the general theory of Dirichlet series during the first decades of the last century was one of the most fashionable topics in analysis (with Bohr's absolute convergence problem very much in its focus), the question whether or not $S=1 / 2$ remained open for a long period. Finally, Bohnenblust and Hille [5] in 1931 in a rather ingenious fashion answered the problem in the positive. They proved (20), and got as a consequence what we now call the Bohr-Bohnenblust-Hille theorem:

$$
S=\frac{1}{2}
$$

Equivalently we see by (47) that

$$
\sup _{D \in \mathscr{H}_{\infty}} \sigma_{a}(D)=\frac{1}{2}
$$

i.e., for each $\varepsilon>0$ and each series $\sum_{n} a_{n} n^{-s} \in \mathscr{H}_{\infty}$ we have $\sum_{n}\left|a_{n}\right| n^{-\frac{1}{2}-\varepsilon}<\infty$, and moreover $\frac{1}{2}$ here can not be improved. A non trivial consequence of (2) is that this supremum is attained.

One of the crucial ideas in the Bohnenblust-Hille approach is that they graduate Bohr's problem: They (at least implicitly) observed that $S_{m}=\frac{1}{M_{m}}$, where

$$
S_{m}=\sup \sigma_{a}(D)-\sigma_{u}(D),
$$

the supremum now taken over all $m$-homogeneous Dirichlet series (recall the definition of $M_{m}$ in (19)). This allows to deduce from (20) the lower bound

$$
S_{m}=\frac{m-1}{2 m}
$$

and hence, since $S_{m} \leq S$, in the limit case as desired $\frac{1}{2} \leq S$. 


\subsection{Main results}

We finally introduce our concept of $\ell_{1}$-multipliers for $\mathscr{H}_{p}$-Dirichlet series. For $1 \leq p \leq \infty$ the image of $H_{p}\left(\mathbb{T}^{\infty}\right)$ under the Bohr transform $\mathfrak{B}$ defined in (4) is denoted by $\mathscr{H}_{p}$. Together with the norm $\|D\|_{\mathscr{C}_{p}}=\left\|\mathfrak{B}^{-1}(D)\right\|_{H_{p}(\mathbb{T} \infty)}$ the vector space of all these so called $\mathscr{H}_{p}$-Dirichlet series $D=\sum_{n} a_{n} n^{-s}$ forms a Banach space. In other words by definition we identify

$$
\mathscr{H}_{p}=H_{p}\left(\mathbb{T}^{\infty}\right) \text {. }
$$

Similarly, we denote by $\mathscr{H}_{p}^{m}$ the image of $H_{p}^{m}\left(\mathbb{T}^{\infty}\right)$ under $\mathfrak{B}$, a closed subspace of $\mathscr{H}_{p}$ (see e.g. [3] and [26]).

Let $\mathscr{E}$ be a set of Dirichlet series (in our setting we typically have $\mathscr{E}=\mathscr{H}_{p}$ or $\left.\mathscr{H}_{p}^{m}\right)$. A sequence $\left(b_{n}\right)$ of complex numbers is said to be an $\ell_{1}$-multiplier for $\mathscr{E}$ whenever

$$
\sum_{n=1}^{\infty}\left|a_{n} b_{n}\right|<\infty
$$

for all $\sum_{n} a_{n} n^{-s} \in \mathscr{E}$. Recall that a sequence $\left(b_{n}\right)$ of complex numbers is said to be multiplicative (or completely multiplicative) whenever $b_{n m}=b_{n} b_{m}$ for all $n, m$.

The Bohr mapping (4) links the concept of multiplicative $\ell_{1}$-multipliers with our previous concept of sets of monomial convergence.

Remark 4.1. Let $\left(b_{n}\right)$ be a multiplicative sequence of complex numbers, and $1 \leq p \leq \infty$. Then $\left(b_{n}\right)$ is an $\ell_{1}$-multiplier for $\mathscr{H}_{p}$ if and only if $\left(b_{p_{k}}\right) \in \operatorname{mon} H_{p}\left(\mathbb{T}^{\infty}\right)$. Clearly, an analogous equivalence holds whenever we replace $\mathscr{H}_{p}$ by $\mathscr{H}_{p}^{m}$.

We now give an almost complete characterization of multiplicative $\ell_{1}$-multipliers of $\mathscr{H}_{p}$-Dirichlet series. The following theorem can be considered as the highlight of this article since it in a very condensed way contains almost all the information given. Recall again that for each bounded sequence $z=\left(z_{n}\right)$ of complex numbers we define

$$
\boldsymbol{b}(z)=\left(\limsup _{n \rightarrow \infty} \frac{1}{\log n} \sum_{j=1}^{n} z_{j}^{* 2}\right)^{1 / 2} .
$$

Theorem 4.2. Let $\left(b_{n}\right)$ be a multiplicative sequence of complex numbers, $1 \leq p<$ $\infty$ and $m \in \mathbb{N}$.

(a) (i) $\left(b_{n}\right)$ is an $\ell_{1}$-multiplier for $\mathscr{H}_{p}^{m}$ if and only if $\left(b_{p_{k}}\right) \in \ell_{2}$.

(ii) $\left(b_{n}\right)$ is an $\ell_{1}$-multiplier for $\mathscr{H}_{\infty}^{m}$ if and only if $\left(b_{p_{j}}\right) \in \ell_{\frac{2 m}{m-1}, \infty}$.

(b) (i) ( $\left.b_{n}\right)$ is an $\ell_{1}$-multiplier for $\mathscr{H}_{p}$ if and only if $\left|b_{p_{j}}\right|<1$ for all $j$ and $\left(b_{p_{k}}\right) \in \ell_{2}$. 
(ii) $\left(b_{n}\right)$ is an $\ell_{1}$-multiplier for $\mathscr{H}_{\infty}$ provided we have that $\left|b_{p_{j}}\right|<1$ for all $j$ and $\boldsymbol{b}\left(\left(b_{p_{j}}\right)\right)<1$.

Conversely, if $\left(b_{n}\right)$ is $\ell_{1}$-multiplier for $\mathscr{H}_{\infty}$, then $\left|b_{p_{j}}\right|<1$ for all $j$ and $\boldsymbol{b}\left(\left(b_{p_{j}}\right)\right) \leq 1$.

For the proof recall the preceding remark and apply Theorems 2.1, 2.2, 3.1, and 3.4 .

\subsection{Bohr's absolute convergence problem - old art in new light}

In what remains we would like to illustrate that this characterization includes many results on the width of Bohr's strips, old and new ones, as special cases:

- The Bohr-Bohnenblust-Hille theorem $S=\frac{1}{2}$ (see (49)) states in terms of multipliers that

$$
\inf \left\{\sigma \mid\left(1 / n^{\sigma}\right) \text { is an } \ell_{1}-\text { multiplier for } \mathscr{H}_{\infty}\right\}=\frac{1}{2} .
$$

Theorem 4.2 -(bii) determines all multiplicative $\ell_{1}$-multiplier for $\mathscr{H}_{\infty}$, and the Bohr-Bohnenblust-Hille theorem is a simple consequence: $S \leq \frac{1}{2}$ since for all $\varepsilon>0$ the sequences $\left(p_{k}^{-\frac{1}{2}-\varepsilon}\right)$ belong to $\mathbf{B}$, and $S \geq \frac{1}{2}$ since $\left(p_{k}^{-\frac{1}{2}+\varepsilon}\right) \notin$ $\overline{\mathbf{B}}$. By (2) this infimum from (53) is attained - a result which in our setting can alternatively be deduced from Theorem 4.2 , bii) since $\left(p_{k}\right)^{-1 / 2} \in \mathbf{B}$.

- For Bohr strips of $m$-homogeneous Dirichlet series we by (52) have that $S_{m}=\frac{m-1}{2 m}$. Again this result can be reformulated into a result on $\ell_{1}$-multipliers for $\mathscr{H}_{\infty}^{m}$ of the type $\left(1 / n^{\sigma}\right)$, and hence it can be easily deduced from the more general Theorem 4.2 - (aii).

- Let now $1 \leq p<\infty$. It is known that each $\mathscr{H}_{p}$-Dirichlet series $D$ has an absolute convergence abscissa $\sigma_{a}(D) \leq 1 / 2$, and that this estimate is optimal:

$$
\sup _{D \in \mathscr{H}_{p}} \sigma_{a}(D)=\frac{1}{2}
$$

This is an $\mathscr{H}_{p}$-analog of (49) (or equivalently (50)) which can be found (implicitly) in [3] and (explicitly) in [2, Theorem 1.1]. After the following reformulation in terms of $\ell_{1}$-multipliers for $\mathscr{H}_{p}$ :

$$
\inf \left\{\sigma \mid\left(1 / n^{\sigma}\right) \text { is an } \ell_{1}-\text { multiplier for } \mathscr{H}_{p}\right\}=\frac{1}{2},
$$


we obtain (54) as an immediate consequence of Theorem 4.2 bi). Note that here in contrast with (53) the infimum in (55) is not attained since $\left(p_{k}^{-1 / 2}\right)_{k} \notin \ell_{2}$ (see also [2] where this was observed for the first time).

- Similarly we obtain $\sup _{D \in \mathcal{H}_{\infty}^{m}} \sigma_{a}(D)=\frac{m-1}{2 m}$ as a consequence of Theorem 4.2 - (aii), and observe that here the infimum corresponding to (55) is attained (see also (25)).

\section{References}

[1] A. Aleman, J.F. Olsen, and E. Saksman. Fatou type theorems on the infinite dimensional polydisk. Preprint 2014.

[2] R. Balasubramanian, B. Calado, and H. Queffélec. The Bohr inequality for ordinary Dirichlet series. Studia Math., 175(3):285-304, 2006.

[3] F. Bayart. Hardy spaces of Dirichlet series and their composition operators. Monatsh. Math., 136(3):203-236, 2002.

[4] F. Bayart, D. Pellegrino, and J. B. Seoane-Sepúlveda. The Bohr radius of the $n$-dimensional polydisk is equivalent to $\sqrt{(\log n) / n}$. Advances in Math., 264:726-746 (2014) Preprint 2014.

[5] H. F. Bohnenblust and E. Hille. On the absolute convergence of Dirichlet series. Ann. of Math. (2), 32(3):600-622, 1931.

[6] H. Bohr. Über die Bedeutung der Potenzreihen unendlich vieler Variablen in der Theorie der Dirichlet-schen Reihen $\sum \frac{a_{n}}{n^{s}}$. Nachr. Ges. Wiss. Göttingen, Math. Phys. Kl., pages 441-488, 1913.

[7] H. Bohr. Über die gleichmäßige Konvergenz Dirichletscher Reihen. J. Reine Angew. Math., 143:203-211, 1913.

[8] B. J. Cole and T. W. Gamelin. Representing measures and Hardy spaces for the infinite polydisk algebra. Proc. London Math. Soc. (3), 53(1):112-142, 1986.

[9] A. M. Davie and T. W. Gamelin. A theorem on polynomial-star approximation. Proc. Amer. Math. Soc., 106(2):351-356, 1989.

[10] R. de la Bretèche. Sur l'ordre de grandeur des polynômes de Dirichlet. Acta Arith., 134(2):141-148, 2008. 
[11] A. Defant, L. Frerick, J. Ortega-Cerdà, M. Ounaïes, and K. Seip. The Bohnenblust-Hille inequality for homogeneous polynomials is hypercontractive. Ann. of Math. (2), 174(1):485-497, 2011.

[12] A. Defant, D. García, and M. Maestre. New strips of convergence for Dirichlet series. Publ. Mat., 54(2):369-388, 2010.

[13] A. Defant, D. García, M. Maestre, and D. Pérez-García. Bohr's strip for vector valued Dirichlet series. Math. Ann., 342(3):533-555, 2008.

[14] A. Defant, M. Maestre, and C. Prengel. Domains of convergence for monomial expansions of holomorphic functions in infinitely many variables. $J$. Reine Angew. Math., 634:13-49, 2009.

[15] S. Dineen. Complex analysis on infinite-dimensional spaces. Springer Monographs in Mathematics. Springer-Verlag London Ltd., London, 1999.

[16] K. Floret. Natural norms on symmetric tensor products of normed spaces. Note Mat., 17:153-188 (1999), 1997.

[17] L. A. Harris. Bounds on the derivatives of holomorphic functions of vectors. In Analyse fonctionnelle et applications (Comptes Rendus Colloq. Analyse, Inst. Mat., Univ. Federal Rio de Janeiro, Rio de Janeiro, 1972), pages 145-163. Actualités Aci. Indust., No. 1367. Hermann, Paris, 1975.

[18] H. Hedenmalm, P. Lindqvist, and K. Seip. A Hilbert space of Dirichlet series and systems of dilated functions in $L^{2}(0,1)$. Duke Math. J., 86(1):1-37, 1997.

[19] H. Helson, and D. Lowdenslager, Prediction theory and Fourier series in several variables. Acta Math., 99:165-202, 1958.

[20] D. Hibert. Gesammelte Abhandlungen (Band 3). Verlag von Julius Springer, Berlin, 1935.

[21] D. Hilbert. Wesen und Ziele einer Analysis der unendlichvielen unabhängigen Variablen. Rend. del Circolo mat. di Palermo, 27:59-74, 1909.

[22] J.-P. Kahane. Some random series of functions, volume 5 of Cambridge Studies in Advanced Mathematics. Cambridge University Press, Cambridge, second edition, 1985.

[23] S. V. Konyagin and H. Queffélec. The translation $\frac{1}{2}$ in the theory of Dirichlet series. Real Anal. Exchange, 27(1):155-175, 2001/02. 
[24] B. Maurizi and H. Queffélec. Some remarks on the algebra of bounded Dirichlet series. J. Fourier Anal. Appl., 16(5):676-692, 2010.

[25] H. Queffélec. H. Bohr's vision of ordinary Dirichlet series; old and new results. J. Anal., 3:43-60, 1995.

[26] H. Queffélec and M. Queffélec. Diophantine approximation and Dirichlet series. HRI Lecture Notes Series, New Delhi 2013.

[27] W. Rudin. Function theory in polydisks. W. A. Benjamin, Inc., New YorkAmsterdam, 1969.

[28] O. Toeplitz. Über eine bei den Dirichletschen Reihen auftretende Aufgabe aus der Theorie der Potenzreihen von unendlichvielen Veränderlichen. Nachrichten von der Königlichen Gesellschaft der Wissenschaften zu Göttingen, pages 417-432, 1913.

[29] F. B. Weissler. Logarithmic Sobolev inequalities and hypercontractive estimates on the circle. J. Funct. Anal., 37(2):218-234, 1980.

[30] P. Wojtaszczyk. Banach spaces for analysts, volume 25 of Cambridge Studies in Advanced Mathematics. Cambridge University Press, Cambridge, 1991.

bayart@math.univ-bpclermont.fr

defant@mathematik.uni-oldenburg.de

frerick@uni-trier.de

maestre@uv.es

psevilla@mat.upv.es 